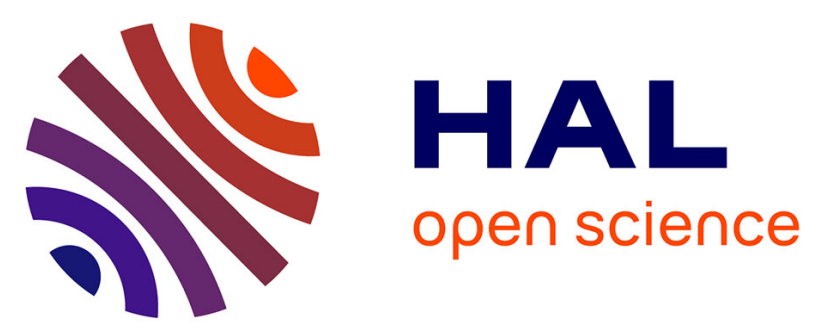

\title{
A combined sensitivity analysis and kriging surrogate modeling for early validation of health indicators
}

Benjamin Lamoureux, Nazih Mechbal, Jean-Rémi Massé

\section{To cite this version:}

Benjamin Lamoureux, Nazih Mechbal, Jean-Rémi Massé. A combined sensitivity analysis and kriging surrogate modeling for early validation of health indicators. Reliability Engineering and System Safety, 2014, 130, pp.12-26. 10.1016/j.ress.2014.03.007 . hal-01059286

\section{HAL Id: hal-01059286 https://hal.science/hal-01059286}

Submitted on 29 Aug 2014

HAL is a multi-disciplinary open access archive for the deposit and dissemination of scientific research documents, whether they are published or not. The documents may come from teaching and research institutions in France or abroad, or from public or private research centers.
L'archive ouverte pluridisciplinaire HAL, est destinée au dépôt et à la diffusion de documents scientifiques de niveau recherche, publiés ou non, émanant des établissements d'enseignement et de recherche français ou étrangers, des laboratoires publics ou privés. 


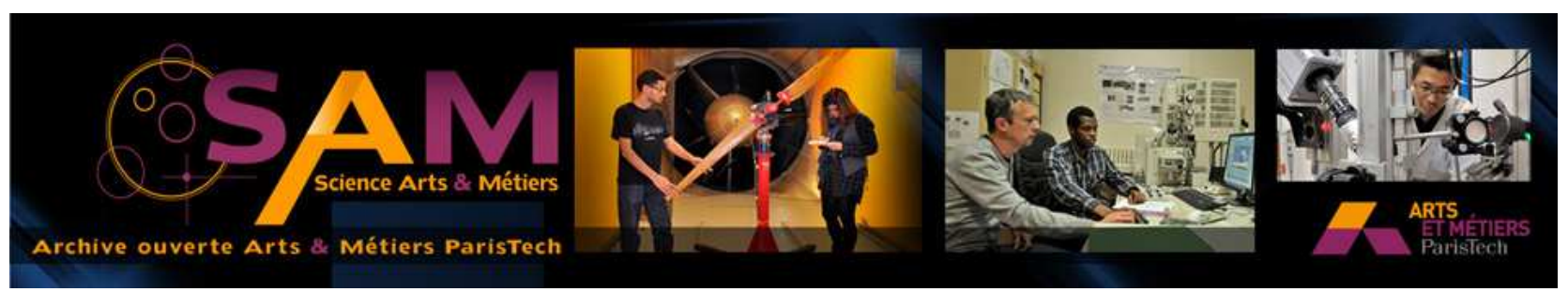

Science Arts \& Métiers (SAM)

is an open access repository that collects the work of Arts et Métiers ParisTech researchers and makes it freely available over the web where possible.

This is an author-deposited version published in: http://sam.ensam.eu

Handle ID: .http://hdl.handle.net/10985/8415

\section{To cite this version :}

Benjamin LAMOUREUX, Nazih MECHBAL, Jean-Rémi MASSÉ - A combined sensitivity analysis and kriging surrogate modeling for early validation of health indicators - Reliability Engineering and System Safety - Vol. 130, p.12-26 - 2014 


\title{
A combined sensitivity analysis and kriging surrogate modeling for early validation of health indicators
}

\author{
Benjamin Lamoureux ${ }^{\mathrm{a}, \mathrm{b}, *}$, Nazih Mechbal ${ }^{\mathrm{a}}$, Jean-Rémi Massé ${ }^{\mathrm{b}}$ \\ ${ }^{a}$ Arts et Métiers ParisTech, PIMM UMR CNRS, Paris, France \\ b Snecma (SAFRAN group), Systems Division, France
}

Keywords:

Health monitoring

Health indicators

Degradation modeling

Validation

Uncertainties propagation

Sensitivity analysis

Surrogate modeling

Kriging

\begin{abstract}
A B S T R A C T
To increase the dependability of complex systems, one solution is to assess their state of health continuously through the monitoring of variables sensitive to potential degradation modes. When computed in an operating environment, these variables, known as health indicators, are subject to many uncertainties. Hence, the stochastic nature of health assessment combined with the lack of data in design stages makes it difficult to evaluate the efficiency of a health indicator before the system enters into service. This paper introduces a method for early validation of health indicators during the design stages of a system development process. This method uses physics-based modeling and uncertainties propagation to create simulated stochastic data. However, because of the large number of parameters defining the model and its computation duration, the necessary runtime for uncertainties propagation is prohibitive. Thus, kriging is used to obtain low computation time estimations of the model outputs. Moreover, sensitivity analysis techniques are performed upstream to determine the hierarchization of the model parameters and to reduce the dimension of the input space. The validation is based on three types of numerical key performance indicators corresponding to the detection, identification and prognostic processes. After having introduced and formalized the framework of uncertain systems modeling and the different performance metrics, the issues of sensitivity analysis and surrogate modeling are addressed. The method is subsequently applied to the validation of a set of health indicators for the monitoring of an aircraft engine's pumping unit.
\end{abstract}

\section{Introduction}

Over the past decade, enhancing dependability has progressively become one of the main challenges for many industries, especially in the field of aeronautics. Indeed, a considerable portion of the average operating expenses of airline companies is attributable to maintenance, repair and overhaul (MRO) and delays and cancellations (D\&C). These expenses are of two types. The first type includes the costs generated by regularly scheduled MRO operations, and the second are those generated by unexpected MRO operations. The expenses associated with the latter can be very high in certain situations, such as when a failure occurs in an isolated, poorly equipped airport. In this situation, additional expenses are generated because of spare parts delivery, aircraft immobilization and passenger indemnification. If the expenses related to regular maintenance are irreducible because they are derived from certification authorities, the other expenses could represent a source of significant savings if one could achieve increased

\footnotetext{
* Corresponding author. Tel.: +33688187030.

E-mail addresses: benjamin.lamoureux@snecma.fr (B. Lamoureux), nazih.mechbal@ensam.eu (N. Mechbal), jean-remi.masse@snecma.fr (J.-R. Massé).
}

dependability. It is for this reason that industries are increasingly more interested in failure anticipation and real-time maintenance strategy optimization.

To predict failures and schedule supervised maintenance, a new field of research, prognostic and health management (PHM), has gradually emerged over the past decade as the unavoidable solution. This new field is receiving much attention from the research community, as evidenced by [1-3] and references therein. PHM is based on the monitoring of relevant variables reflecting the different degradation modes likely to occur in the system. These relevant variables are termed health indicators (HIs). A classical PHM framework usually performs detection, identification and prognostic. While different forms of the PHM process can be found, the most commonly used, at least in the industry, is the openstructure architecture for conditioned based maintenance (OSACBM) scheme [4]. Although PHM is a quite recent discipline, it has reached a certain maturity with the development of its own standards, as shown in $[5,6]$. It has also been frequently applied and has demonstrated good results, first in its original field of application, structural health monitoring (SHM) [7], and later, in other fields, such as bearing monitoring [8] and battery life prediction [9]. The present work is dedicated to the monitoring of 


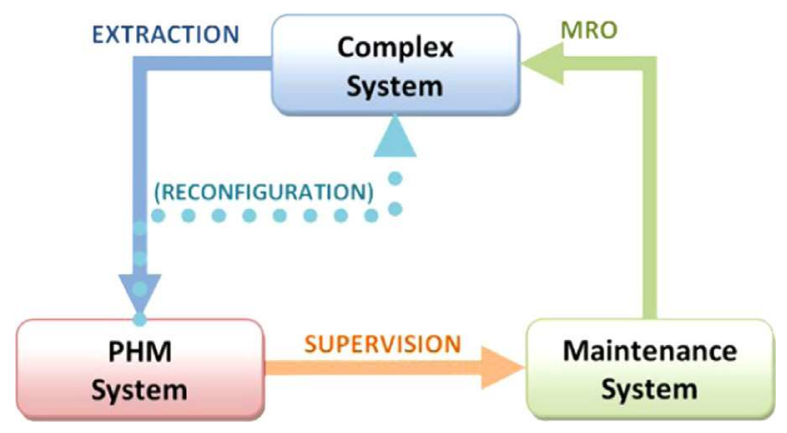

Fig. 1. Interaction scheme between the complex system, the PHM system and the maintenance system.

multi-physics complex systems with closed loop control, which is a novel and relatively unexplored application of PHM.

A PHM system can be defined as an entity interacting, on the one hand, with the complex system via an extraction process and, on the other hand, with the maintenance system via a supervision process (see Fig. 1). The purpose of the extraction process is to provide the set of HIs to the PHM system. The purpose of the supervision process is to assess the current health status of the complex system, to predict its evolution and to propose corrective or predictive actions to maintenance operators.

Whereas the supervision framework is the subject of many papers, the extraction framework is rarely addressed because its complexity is often underestimated. Indeed, at first glance, the extraction simply consists of recording data, but the real difficulty is to determine which data are to be recorded. Even if some research has been conducted to define certain generic methods for constructing HIs, such as parity space [10], most of these methods are not adapted to overcome certain challenges, such as uncertainties, imposed sensor numbers and locations, limited computation capabilities and prohibitive controller retrofit costs [11]. Thus, when an actual system is considered, it is necessary to perform a complete knowledge analysis to determine critical degradation modes and to construct relevant physics based HIs that are compatible with the sensor's configuration and the computation capabilities. These HIs also must be validated before the system enters into service because of the controller retrofit costs. This last point is the most critical because PHM processes are inherently stochastic problems, and it is obviously difficult to validate something stochastic before the availability of measured in-service data.

To overcome this lack of data for the validation of HIs, numerical modeling associated with a complete management of parameters uncertainties [12] is used during design stages to simulate the HIs distributions with and without degradations. This operation requires a good knowledge of input uncertainties, which is usually acquired through expertise and experience feedback from similar systems. Once both the healthy and faulty distributions of HIs are computed, some numerical key performance indicators (NKPIs) are computed to quantify the quality of the $\mathrm{HI}$ set in terms of detection, identification and prognostic potential. In the aeronautic industry, the NKPIs could account for a major step forward as online data recording is very expensive.

However, the propagation of uncertainties presents two major issues. First, in cases where the physics-based model is defined by numerous parameters, the quantification of uncertainties can rapidly become very time-demanding and expensive because it needs to collect much knowledge from various sources. Then, when the simulation runtime of the physics-based model is important, for example, several minutes or hours, the computation time required for uncertainties propagation becomes prohibitive. This is all the more true as the PHM system is composed of numerous HIs and numerous degradation modes. This paper proposes to use a combination of sensitivity analysis techniques and kriging surrogate modeling to solve both issues. Sensitivity analysis is performed in two stages. First, after having roughly determined the variation range of parameters, the Morris method is used to achieve a reduction in the parameters' space dimension. This allows for determining the set of uncertain parameters that will be the inputs of the kriging model. The computation of Sobol indices is then performed to hierarchically sort the uncertain parameters with respect to their effects on outputs. From this hierarchization, we identify the most influent parameters on which the fine uncertainties quantification are targeted. Kriging is used to obtain a low computational cost function for estimating the model outputs. Due to the reduction of input space provided by the sensitivity analysis, the size of the learning design of experiments is significantly reduced. Finally, both the computation of Sobol indices and uncertainties propagation can be run on the kriging model at reasonable computation time costs. Finally, the efficiency of the whole method is tested on a real complex system, namely, the pumping unit of an aircraft engine fuel system.

The remainder of the paper is organized as follows: In Section 2 , the background of uncertain systems modeling are addressed through specific definitions of key terms. The numerical key performance indicators for $\mathrm{HI}$ validation in design stages are then introduced. The Sections 3 and 4, respectively, are dedicated to the sensitivity analysis and the surrogate modeling. Finally, Section 5 introduces the application system, and Section 6 presents and discusses the results.

\section{Uncertain systems modeling}

In [13], uncertainty is defined as "the incompleteness in knowledge and the inherent variability of the system and its environment". In this section, the modeling of a complex system $\subseteq$ accounting for uncertainties is addressed through specific definitions of key terms

\subsection{System modeling}

\subsubsection{Numerical model}

We propose to represent the determinist model of a complex system by the function $f$ :

$\boldsymbol{Y}=f\left(\boldsymbol{U}, \rho_{1}, \ldots, \rho_{p}\right)$

where $\boldsymbol{U}$ is the matrix of the model inputs, $\boldsymbol{Y}$ is the matrix of the model outputs and $\rho_{1}, \ldots, \rho_{p}$ are the model parameters. As the numerical model is a discrete system, considering a sample period equal to $T$ and a simulation of $k$ samples, the input and output matrix is written as follows:

$$
\begin{aligned}
& \boldsymbol{U}=\left|\begin{array}{ccc}
u_{1}(0) & \ldots & u_{n}(0) \\
u_{1}(T) & \ldots & u_{n}(T) \\
\vdots & \ddots & \vdots \\
u_{1}((k-1) T) & \ldots & u_{n}((k-1) T) \\
u_{1}(k T) & \ldots & u_{n}(k T)
\end{array}\right| \in \mathbb{R}^{k \times n} ; \\
& \boldsymbol{Y}=\left|\begin{array}{ccc}
y_{1}(0) & \ldots & y_{m}(0) \\
y_{1}(T) & \ldots & y_{m}(T) \\
\vdots & \ddots & \vdots \\
y_{1}((k-1) T) & \ldots & y_{m}((k-1) T) \\
y_{1}(k T) & \ldots & y_{m}(k T)
\end{array}\right| \in \mathbb{R}^{k \times m}
\end{aligned}
$$




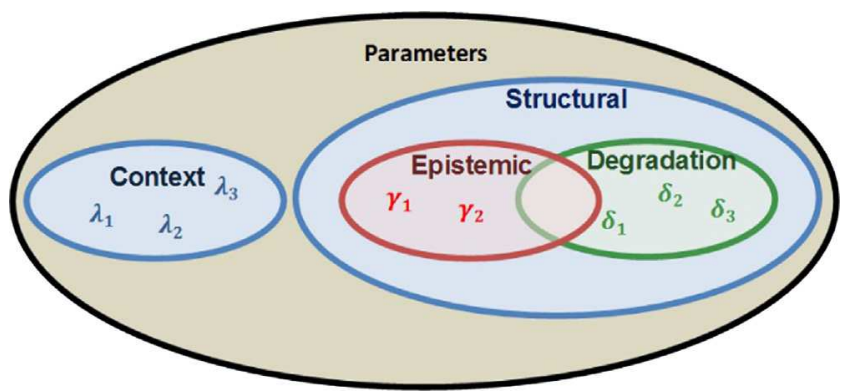

Fig. 2. Classification of the different types of parameters for system modeling. In this example, the system is modeled from 3 context parameters, 2 epistemic parameters and 3 degradation parameters.

\subsubsection{Parameters}

Parameters are variables that are considered constant during a single simulation but can vary between two different runs. When a variable is not constant during a run, it is classified as an input.

We propose the following formalism. Parameters $\rho_{1}, \ldots, \rho_{p}$ are divided into two different types: the context parameters $\lambda_{1}, \ldots, \lambda_{c}, c \leq p$ and the structural parameters $\beta_{1}, \ldots, \beta_{s}, s \leq p$. As a parameter cannot be of both a context and structural type, $s+c=p$. The structural parameters are sub-divided into epistemic parameters $\gamma_{1}, \ldots, \gamma_{e}, e \leq s$ and degradation parameters $\delta_{1}, \ldots$, $\delta_{d}, d \leq s$. A parameter can be simultaneously epistemic and degradation (see Fig. 2). For example, consider a hydraulic model defined by the following parameters: cylinder diameter $D_{c y l}$, pump displacement $D i s_{\text {pump }}$, pump leakage diameter $D_{\text {leak }}$,ambient temperature $T_{a m b}$, fluid temperature $T_{\text {fluid }}$. Following our formalism, $T_{a m b}$ and $T_{\text {fluid }}$ are context parameters, $D_{c y l}$ and Dis $s_{\text {pump }}$ are epistemic parameters and $D_{\text {leak }}$ is a degradation parameter.

To formalize in a more mathematical way the objects that we handle during the modeling of the system, we propose to define the following spaces.

\subsubsection{Parameterization}

We propose to define the parameter space $P$ as a Euclidian vector space of dimension $p$ provided with canonical base $\left(\boldsymbol{e}_{1}^{\mathbf{P}}, \ldots, \boldsymbol{e}_{\boldsymbol{p}}^{\mathbf{P}}\right)^{T}$ and Euclidian norm $\|\cdot\| \mathbf{p}$. Parameterization $\boldsymbol{\rho}$ is defined as a vector of $\mathcal{P}$ whose components are the parameters' values: $\boldsymbol{\rho}=\rho_{1} \boldsymbol{e}_{1}^{\mathbf{P}}+\cdots+\rho_{p} \boldsymbol{e}_{\boldsymbol{p}}^{\mathbf{P}}=\left(\rho_{1}, \ldots, \rho_{p}\right)^{T}$. Nominal parameterization $\boldsymbol{\rho}^{\text {nom }}$ is defined for the nominal values of the parameters as $\boldsymbol{\rho}^{\text {nom }}=\left(\rho_{1}^{\text {nom }}, \ldots, \rho_{p}^{\text {nom }}\right)^{T}$.

\subsubsection{Configuration}

We propose to define the configuration space $\mathscr{E}$ as a subspace of $\mathscr{P}$ of dimension $e$ with inherited canonical base $\left(\boldsymbol{e}_{1}^{\mathscr{E}}, \ldots, \boldsymbol{e}_{\boldsymbol{e}}^{\mathscr{E}}\right)^{T}$ and Euclidian norm $\|\cdot\|_{\varepsilon}$. Configuration $\gamma$ is defined as a vector of $C$ whose components are the epistemic parameters' values: $\gamma=$ $\gamma_{1} \boldsymbol{e}_{1}^{\mathscr{E}}+\cdots+\gamma_{e} \boldsymbol{e}_{\boldsymbol{e}}^{\mathscr{E}}=\left(\gamma_{1}, \ldots, \gamma_{e}\right)^{T}$. Nominal configuration $\boldsymbol{\gamma}^{\text {nom }}$ is defined for the nominal values of the epistemic parameters as $\gamma^{\text {nom }}=\left(\gamma_{1}^{\text {nom }}, \ldots, \gamma_{e}^{\text {nom }}\right)^{T}$.

\subsubsection{Context}

We propose to define the context space $C$ as a subspace of $\mathscr{P}$ of dimension $c$ with inherited canonical base $\left(\boldsymbol{e}_{1}^{C}, \ldots, \boldsymbol{e}_{\boldsymbol{c}}^{C}\right)^{T}$ and Euclidian norm $\|\cdot\|_{\mathcal{K}}$. Context $\lambda$ is defined as a vector of $C$ whose components are the context parameters' values: $\lambda=\lambda_{1} \boldsymbol{e}_{1}^{C}+\cdots+\lambda_{c} \boldsymbol{e}_{\boldsymbol{c}}^{C}=$ $\left(\lambda_{1}, \ldots, \lambda_{c}\right)^{T}$. Nominal context $\lambda^{\text {nom }}$ is defined for the nominal values of the context parameters as $\lambda^{\text {nom }}=\left(\lambda_{1}^{\text {nom }}, \ldots, \lambda_{c}^{\text {nom }}\right)^{T}$.

\subsubsection{Condition}

We propose to define the condition space $\mathscr{D}$ as a subspace of $\mathscr{P}$ of dimension $d$ with inherited canonical base $\left(\boldsymbol{e}_{1}^{\mathscr{D}}, \ldots, \boldsymbol{e}_{\boldsymbol{d}}^{\mathcal{D}}\right)^{T}$ and Euclidian norm $\|\cdot\|_{Q}$. Condition $\delta$ is defined as a vector of $\mathcal{D}$ whose components are the degradation parameters' values: $\boldsymbol{\delta}=$ $\delta_{1} \boldsymbol{e}_{1}^{D}+\cdots+\delta_{d} \boldsymbol{e}_{\boldsymbol{d}}^{\mathscr{D}}=\left(\delta_{1}, \ldots, \delta_{d}\right)^{T}$. Nominal condition $\boldsymbol{\delta}^{\boldsymbol{n o m}}$ is defined for the nominal values of the degradation parameters as $\delta^{\text {nom }}=\left(\delta_{1}^{\text {nom }}, \ldots, \delta_{d}^{\text {nom }}\right)^{T}$.

\subsubsection{Degradation}

We propose to define the degradation space $O$ as an affine subspace of $\mathscr{D}$ with origin $\Delta^{\text {nom }}$ and the same dimension, base and norm. Degradation $\boldsymbol{\omega}$ is a vector of $O$. Degradation $\boldsymbol{\omega}=\omega_{1} \boldsymbol{e}_{1}^{O}+\cdots$ $+\omega_{d} \boldsymbol{e}_{\boldsymbol{d}}^{O}=\left(\omega_{1}, \ldots, \omega_{d}\right)^{T}$ is defined by its direction (or mode) and its magnitude. Degradation mode is the unitary vector $\boldsymbol{\omega} /\|\boldsymbol{\omega}\|_{O}$ of the same direction and sense as $\omega$, and the magnitude is the norm $\|\boldsymbol{\omega}\|_{O}$ of $\boldsymbol{\omega}$ in $O$. It can be verified that if $\boldsymbol{\omega}=0$, then the system is in its nominal condition. In this paper, as only single degradation modes are considered, there are $d$ degradation modes defined as the vectors of the canonical base. In this case, a degradation can be written as $\boldsymbol{\omega}=\omega \boldsymbol{e}_{\boldsymbol{j}}^{O}$, where $\omega$ is the degradation magnitude and $\boldsymbol{e}_{\boldsymbol{j}}^{O}$ is the degradation mode.

The system cannot be robust to all the degradation modes' magnitudes because, at some point, a failure will appear. The maximal admissible magnitude (MAM) of a degradation mode is defined as the magnitude for which this failure occurs and is written as $\omega_{M A M}^{j}$ for degradation mode $\boldsymbol{e}_{\boldsymbol{j}}^{O}$, and the maximal degradation is written as $\omega_{\max }^{j}=\omega_{M A M}^{j} \boldsymbol{e}_{j}^{O}$.

\subsubsection{Syndromes}

As previously stated, HIs are relevant variables that provide some information about the health status of a given system. They are named $\varphi_{1}, \ldots, \varphi_{h}$. We propose to define the syndrome space $S$ as a Euclidian vector space of dimension $h$ provided with canonical base $\left(\boldsymbol{b}_{1}^{S}, \ldots, \boldsymbol{b}_{\boldsymbol{h}}^{S}\right)^{T}$ and Euclidian norm $\|\cdot\|_{S}$. Syndrome $\boldsymbol{\varphi}$ is defined as a vector of $S$ whose components are the HI values $\boldsymbol{\varphi}=\varphi_{1} \boldsymbol{b}_{1}^{S}+\cdots+\varphi_{h} \boldsymbol{b}_{\boldsymbol{h}}^{S}=\left(\varphi_{1}, \ldots, \varphi_{h}\right)^{T}$. A syndrome is computed from the output matrix of the model by an extraction function $g$ defined as follows:

$\boldsymbol{\varphi}=g(\boldsymbol{Y})$

Nominal syndrome $\boldsymbol{\varphi}^{\text {nom }}$ is defined for the nominal values of the HI as $\varphi^{\text {nom }}=\left(\varphi_{1}^{\text {nom }}, \ldots, \varphi_{h}^{\text {nom }}\right)^{T}$. The nominal condition is the image of the nominal parameterization by the extraction function:

$\boldsymbol{\varphi}^{\text {nom }}=g\left(f\left(\boldsymbol{U}, \beta_{1}^{\text {nom }}, \ldots, \beta_{p}^{\text {nom }}\right)\right)$

By defining $h=g_{0} f$, the numerical model can be written as $\boldsymbol{\varphi}=h\left(\boldsymbol{U}, \boldsymbol{\gamma}, \boldsymbol{\lambda}, \boldsymbol{\delta}^{\boldsymbol{n o m}}+\boldsymbol{\omega}\right)$

A syndrome $\boldsymbol{\varphi}$ is defined by its direction $\boldsymbol{\varphi} /\left\|\boldsymbol{\varphi}_{S}\right\|$ and its intensity $\left\|\boldsymbol{\varphi}_{S}\right\|$. The diagnostic problem can be regarded as an identification of function $h$. To ensure identification and to ensure that one syndrome corresponds to only one degradation mode, a good property for function $h$ is injectivity.

\subsection{Uncertainties management}

\subsubsection{Uncertainties quantification}

For the modeling of multi-physics complex systems subject to actual operating conditions, managing the uncertainties of the parameters is of paramount importance. In this paper, two types 


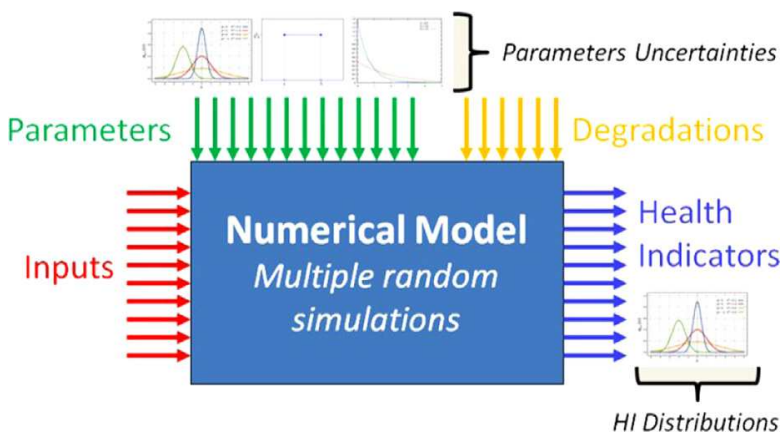

Fig. 3. Numerical model with parameter uncertainties and degradations.

of uncertainties are considered: random uncertainties derived from environment variations affecting context and systematic uncertainties derived from manufacturing variations affecting configurations. Taking into account uncertainties requires replacing some of the deterministic parameters $\lambda_{i}, i \subset[1 ; c]$ and $\gamma_{j}, j \subset[1 ; e]$ of the model with random variables $\Lambda_{i}$ and $\Gamma_{j}$. These random variables can be characterized by their probability density function (PDF). Uncertainties localization consists of identifying the parameters subject to uncertainties, that is, the uncertain parameters. Uncertainties quantification involves determining the PDF for every uncertain parameter, which usually is the result of expertise and experience feedback on similar systems. The PDFs are usually defined by the type of their distribution (normal, uniform, generalized extreme values, etc.) and their parameter vector $\boldsymbol{\theta}=\left(\theta_{1}, \ldots, \theta_{r}\right)^{T}$ where $r$ represents the number of parameters for the considered type of distribution. For example, $\Lambda_{3} \sim G^{\mathscr{E} V} v(\mu, \sigma, \xi)$ means that the uncertainty on $\Lambda_{3}$ follows a generalized extreme value law of location $\mu$, scale $\sigma$ and shape $\xi$. The uncertainties quantification can be a very expensive step when the number of parameters is large. Section 5 will discuss how to reduce the costs associated with sensitivity analysis methods.

\subsubsection{Uncertainties propagation}

Knowing the uncertain parameters, it is possible to compute stochastic HI distributions from a deterministic model by randomly sampling them according to their PDFs (see Fig. 3). This operation is called uncertainties propagation [12]. While many tools are available, the most common is the Monte-Carlo simulation [14]. This method is used in this paper with a number of iterations equal to $q$. In this probabilistic framework, (5) can be written as

$\Phi=h\left(\boldsymbol{U}, \boldsymbol{\Gamma}, \boldsymbol{\gamma}^{\prime}, \boldsymbol{\Lambda}, \lambda^{\prime}, \boldsymbol{\delta}^{\mathbf{n o m}}+\boldsymbol{\omega}\right)$

where $\boldsymbol{\Phi}=\left(\Phi_{1}, \ldots, \Phi_{h}\right)$ is the random vector of HIs, $U$ is the input matrix, $\boldsymbol{\Gamma}=\left(\boldsymbol{\Gamma}_{1}, \ldots, \boldsymbol{\Gamma}_{a}\right)$ is the random vector of uncertain epistemic parameters, $\boldsymbol{\gamma}^{\prime}=\left(\gamma_{1}, \ldots, \gamma_{e-a}\right)$ is the determinist vector of fixed epistemic parameters, $\boldsymbol{\Lambda}=\left(\boldsymbol{\Lambda}_{1}, \ldots, \boldsymbol{\Lambda}_{b}\right)$ is the random vector of uncertain context parameters, $\lambda^{\prime}=\left(\lambda_{1}, \ldots, \lambda_{c-b}\right)$ is the determinist vector of fixed context parameters, $\boldsymbol{\delta}^{\mathbf{n o m}}$ is the determinist nominal condition and $\omega$ is a determinist degradation.

\subsubsection{Syndrome distributions}

Let us consider $q$-samples $\gamma_{(\boldsymbol{q})}=\left(\gamma_{k 1}, \ldots, \gamma_{k e}\right)_{k=1, \ldots, q}$ and $\lambda_{(\boldsymbol{q})}=$ $\left(\lambda_{k 1}, \ldots, \lambda_{k c}\right)_{k=1, \ldots, q}$, realizations of random vectors $\boldsymbol{\Gamma}$ and $\boldsymbol{\Lambda}$. By using (6), it is possible to compute $\boldsymbol{\varphi}_{(\boldsymbol{q})}=\left(\varphi_{k 1}, \ldots, \varphi_{k h}\right)_{k=1, \ldots, q}$, the realization of random vector $\boldsymbol{\Phi}$, for different degradations $\boldsymbol{\omega}$. Syndrome distribution is defined as the $q \times h$ matrix $\boldsymbol{S}^{\boldsymbol{\omega}}$ dependent on degradation $\boldsymbol{\omega}$ and containing the values of $\boldsymbol{\varphi}_{(\boldsymbol{q})}$. For example, the healthy distribution is $\boldsymbol{S}^{0}$, and the faulty distribution of degradation mode $\boldsymbol{e}_{\boldsymbol{j}}^{O}$ with magnitude $\omega$ is $\boldsymbol{S}^{\omega \boldsymbol{e}_{j}^{o}}$.

In the case of systems actually marketed, it is necessary to make a distinction between individual syndrome distributions (ISDs) and fleet syndrome distributions (FSDs). The former is computed by propagating only random uncertainties, i.e., with $\boldsymbol{\Gamma}$ a deterministic vector with nominal values in (6). The latter is computed by propagating both random and systematic uncertainties. The ISDs traduce on the variability between different missions on the same system, whereas the FSDs traduce the variability between different systems of the same type. Even if the FSDs contain more information, the ISDs are useful when uncertainties are too important to ensure required detection performances. Actually, computing ISDs and FSDs allows choosing between the generalized monitoring of a group of systems and the particularized monitoring of a single system. For example, the healthy FSD is written as $\boldsymbol{S}_{F}^{0}$, and the ISD of degradation $\boldsymbol{\omega}$ is written as $\boldsymbol{S}_{I}^{\boldsymbol{\omega}}$.

\subsubsection{Reduced syndromes}

From the syndrome distributions, it is possible to estimate the parameter vector of the HIs' PDFs. This estimation is performed by maximum likelihood estimation (MLE) from prior knowledge of the distribution type. The MLE of the PDF parameter vector of the syndrome distribution $\boldsymbol{S}_{F}^{\boldsymbol{\omega}}$ is $\boldsymbol{\theta}_{F}^{\boldsymbol{\omega}} \in \mathbb{R}^{\boldsymbol{h} \times \boldsymbol{r}}$ with $\boldsymbol{r} \in \mathbb{N}$ the number of parameters for the selected PDF type. For example, the healthy reduced syndrome for normal PDF selection is $\boldsymbol{\theta}_{F}^{0} \in \mathbb{R}^{\boldsymbol{h} \times 2}$.

\section{Numerical key performance indicators}

The purpose of this section is to propose definitions for numerical key performance indicators (NKPIs). These NKPIs are metrics aimed at performing early quantification of an HI set efficiency in design phases. As they can be computed for both fleet model and individual model, in this section, type can be replaced either by $F$ or $I$.

\subsection{Detection NKPIs}

Typically, detection specifications yield a maximum false positive rate (FP) and a minimum false negative rate (FN) for detection. The basis of detection theory can be found in [15]. The detection NKPIs are based on receiver operating characteristic (ROC) curves [16]. Two types of detection NKPIs are used: a global detectability matrix and a compliant detectability matrix. The ROC curve between two PDFs of parameter vectors $\boldsymbol{\theta}$ and $\boldsymbol{\theta}^{\prime}$ is written as $\operatorname{ROC}\left(\boldsymbol{\theta}, \boldsymbol{\theta}^{\prime}\right)$. For a given couple HI $i$ and degradation $\boldsymbol{\omega}$, the ROC curve between healthy and faulty PDF parameters is computed from the reduced syndromes asROC $\left(\boldsymbol{\theta}_{\text {type }}^{0}(i,:), \boldsymbol{\theta}_{\text {type }}^{\omega}(i,:)\right)$, where $\boldsymbol{\theta}(\boldsymbol{i},:)$ is the $i$ th line of matrix $\boldsymbol{\theta}$. Healthy and faulty PDF parameters are the estimated parameters of, respectively, the healthy distribution and the faulty distribution. The healthy distribution is obtained by propagating uncertainties on a model with no degradation mode. The faulty degradation is obtained via the repetition of this uncertainties propagation where each time a new degradation mode is modeled. In practice, we suppose that these distributions are of generalized extreme value type, and we estimate the parameters via maximum likelihood.

\subsubsection{Global detectability}

For a given ROC curve, global detectability (GD) is defined as a function calculated from the area under the curve (AUC) [17]. The closer to one the value is, the higher the detection potential. As GD does not depend on the detection specifications, it is robust to 


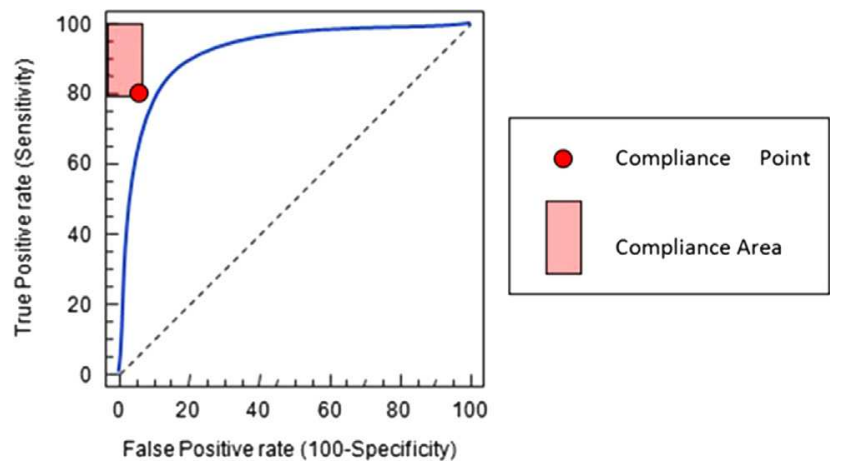

Fig. 4. Compliance point and compliance area on a ROC curve.

specification changes, and it is equivalent to the Gini coefficient [18].

$G D\left(\boldsymbol{\theta}, \boldsymbol{\theta}^{\prime}\right)=2 \times A U C\left(R O C\left(\boldsymbol{\theta}, \boldsymbol{\theta}^{\prime}\right)\right)-1$

GD is computed for each couple $\left(\boldsymbol{\theta}_{\text {type }}^{0}(i,:), \boldsymbol{\theta}_{\text {type }}^{\omega_{\max }^{j}}(i,:)\right)$, $(i, j) \in[1 ; \mathrm{h}] \times[1 ; \mathrm{d}]$ and the following NKPI, the global detectability matrix $\mathbf{G D x}$, is constructed:

$\boldsymbol{G D x}=\left|\begin{array}{ccc}G D\left(\boldsymbol{\theta}_{\text {type }}^{0}(1,:), \boldsymbol{\theta}_{\text {type }}^{\omega_{\max }^{1}}(1,:)\right) & \ldots & G D\left(\boldsymbol{\theta}_{\text {type }}^{0}(1,:), \boldsymbol{\theta}_{\text {type }}^{\omega_{\max }^{d}}(1,:)\right) \\ \vdots & \ddots & \vdots \\ G D\left(\boldsymbol{\theta}_{\text {type }}^{0}(h,:), \boldsymbol{\theta}_{\text {type }}^{\omega_{\max }^{1}}(h,:)\right) & \ldots & G D\left(\boldsymbol{\theta}_{\text {type }}^{0}(h,:), \boldsymbol{\theta}_{\text {type }}^{\omega_{\max }^{d}}(h,:)\right)\end{array}\right| \in[0 ; 1]^{h \times d}$

\subsubsection{Compliant detectability}

For a given ROC curve, the compliance point is defined as the point of the coordinates (FPspec, TPspec) with FPspec as the specified maximal false positive rate and TPspec as the specified minimal true positive rate delimiting the compliance area (cf. Fig. 4). Compliant detectability (CD) is defined as follows:

$C D\left(\boldsymbol{\Theta}, \boldsymbol{\Theta}^{\prime}\right)=\left\{\begin{array}{l}1 \text { if } \operatorname{ROC}\left(\boldsymbol{\Theta}, \boldsymbol{\Theta}^{\prime}\right) \text { is above the compliance point } \\ 0 \text { if } \operatorname{ROC}\left(\boldsymbol{\Theta}, \boldsymbol{\Theta}^{\prime}\right) \text { is under the compliance point }\end{array}\right.$

If specifications on the FP are considerably restrictive, it is difficult to observe the compliance point on the curve. In this case, it is possible to use the semi-logarithmic ROC curve with a logarithmic scale in abscissa for the FP to give more clarity to the curve. Eventually, CD is computed for each couple $\left(\boldsymbol{\theta}_{\text {type }}^{0}(i,:)\right.$, $\left.\boldsymbol{\theta}_{\text {type }}^{\boldsymbol{\omega}_{\max }^{d}}(i,:)\right),(i, j) \in[1 ; h] \times[1 ; d]$ and the following NKPI, the compliant detectability matrix $\mathbf{C D x}$ is constructed:

$\boldsymbol{C D} \boldsymbol{x}=\left|\begin{array}{ccc}C D\left(\boldsymbol{\theta}_{\text {type }}^{0}(1,:), \boldsymbol{\theta}_{\text {type }}^{\omega_{\max }^{1}}(1,:)\right) & \ldots & C D\left(\boldsymbol{\theta}_{\text {type }}^{0}(1,:), \boldsymbol{\theta}_{\text {type }}^{\omega_{\max }^{d}}(1,:)\right) \\ \vdots & \ddots & \vdots \\ C D\left(\boldsymbol{\theta}_{\text {type }}^{0}(h,:), \boldsymbol{\theta}_{\text {type }}^{\omega_{\max }^{1}}(h,:)\right) & \ldots & C D\left(\boldsymbol{\theta}_{\text {type }}^{0}(h,:), \boldsymbol{\theta}_{\text {type }}^{\omega_{\max }^{d}}(h,:)\right)\end{array}\right| \in\{0,1\}^{h \times d}$

\subsection{Identification NKPIs}

The classical identification process aims at finding the most probable degradation mode of the system, and as such, it is based on the classification of the current reduced syndromes relative to a reference database of different reduced syndromes corresponding to the degradations. In this section, one identification NKPI is defined based on the signature vectors: the cross identificability matrix.

\subsubsection{Signature and distinguishability}

The signature space $\mathscr{R}$ is defined as a Euclidian vector space of dimension $h$ provided with canonical base $\left(\mathbf{g}_{1}^{\mathscr{R}}, \ldots, \mathbf{g}_{\boldsymbol{p}}^{\mathscr{R}}\right)^{T}$ and norm $\|\cdot\|_{\mathscr{R}}$. The signature $\boldsymbol{S g \boldsymbol { n } ^ { j }}$ of a degradation mode $j$ is a vector of $\mathscr{R}$ indicating the level of similarity between the reduced healthy syndrome and the reduced faulty syndrome computed for the MAM. As it is a function of the global detectability, the sign of the difference between distributions means:

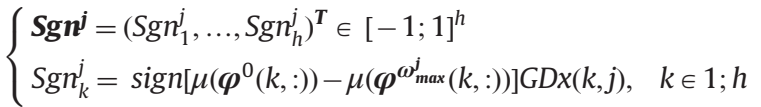

Thus, the distinguishability index Dis is defined as the angle between two similar vectors. For two degradation modes $\mathrm{j}$ and $\mathrm{k}$,

$\left.\operatorname{Dis}\left(\boldsymbol{S g} n^{j}, \boldsymbol{S g} n^{k}\right)=\arccos \frac{S g n^{j T} \cdot S g n^{k}}{S g n_{\mathscr{R}}^{j} S g n_{\mathscr{R}}^{k}}\right)$

\subsubsection{Cross identificability}

The cross identificability matrix $\mathbf{C l \boldsymbol { x }}$ is a symmetric matrix defined as follows:

$\boldsymbol{C I x}=\left|\begin{array}{ccc}\operatorname{Dis}\left(\boldsymbol{S g n}^{1}, \boldsymbol{S g n}^{1}\right) & \ldots & \operatorname{Dis}\left(\boldsymbol{S g n} \boldsymbol{k}^{\boldsymbol{k}}, \boldsymbol{S g n}^{1}\right) \\ \vdots & \ddots & \vdots \\ \operatorname{Dis}\left(\boldsymbol{S g n}^{1}, \boldsymbol{S g n}^{k}\right) & \ldots & \operatorname{Dis}\left(\boldsymbol{S g n}^{\boldsymbol{k}}, \boldsymbol{S g n}^{\boldsymbol{k}}\right)\end{array}\right| \in \mathbb{R}^{d \times d}$

\subsection{Prognostic NKPIs}

\subsubsection{Minimal detectable magnitude}

The minimal detectable magnitude (MDM) of degradation mode $j$ is $\omega_{M D M}^{j}$ and is defined as follows:

$\omega_{M D M}^{j}=\min _{\omega \in \mathbb{R}, i \in 1 ; h}\left\{\omega / C D\left(\boldsymbol{\theta}_{\text {type }}^{0}(i,:), \boldsymbol{\theta}_{\text {type }}^{\omega \boldsymbol{e}_{j}^{o}}(i,:)\right)=1\right\}$

It is the lowest magnitude for which a degradation mode is detectable when all HIs are combined. If the ensemble defined in Eq. (16) is empty, then $\omega_{M D M}^{j}=\varnothing$ and the degradation mode is not detectable.

\subsubsection{Detection margin}

To quantify the prognostics capabilities, the following prognostic NKPI is defined as the detection margin vector $\mathbf{D M v}$, which is computed as follows:

$\boldsymbol{D M} \boldsymbol{v}=\left(\omega_{M D M}^{1}-\omega_{M A M}^{1}, \ldots, \omega_{M D M}^{d}-\omega_{M A M}^{d}\right) \in \mathbb{R}^{d}$

The detection margin vector indicates, for each degradation mode, the gap between the lowest detectable magnitude and the highest admissible magnitude before degradation.

Due to these NKPIs, it is possible to determine the detectable degradation modes, to determine whether they are separable and to determine the margin between the detection magnitude and the failure magnitude. It is also possible to quantify the efficiency of each single $\mathrm{HI}$ for the detection and the identification process such that useless HIs would be eliminated.

\section{Sensitivity analysis}

As previously mentioned, the computation of the necessary data for the validation of HIs is based on uncertainties propagation. The quality of this propagation depends on the quantification of uncertainties. This task can be very long and complex when the number of parameters is large and no priorities are defined. Thus, to enhance the quantification of uncertainties, we propose to use sensitivity analysis techniques. Sensitivity analysis (SA) is the study of how the uncertainties in its inputs. In the present application, inputs are parameters and outputs are HIs. There are three types of sensitivity analysis methods: local, global and screening [19]. In this paper, we will use one screening technique, 
the Morris method [20], and one global technique, the computation of Sobol indices [21].

\subsection{Morris method}

The Morris method belongs to the screening techniques family. These techniques are used to perform a fast but rather coarse exploration of the behaviors of outputs of a costly computation cost with numerous inputs, typically hundreds. Screening methods are based on a discretization of the inputs into different levels, they do not use probabilities. Many types of screening methods are described in literature, such as the supersaturated design, group screening or sequential bifurcation. The usual design of experiments (DOE), such as factorial designs or one at a time (OAT) designs, are also part of this family. In this paper, the Morris screening method is used. The Morris method consists in randomly repeating $r$ times an OAT design in the input space. This method provides a sorting of inputs into three categories:

- Inputs with negligible effects

- Inputs with linear effects without interaction

- Inputs with non-linear effects and/or interactions

Each repetition $i(i=1, \ldots, r)$ evaluates an elementary effect $E_{j}^{(i)}$ (increase between two successive points) for each input $X_{j}$. The $r$ repetitions of the DOE furnishes an $r$-sample of the effects for each input $X_{j}$ from which the sensitivity indices are as follows:

$\mu_{j}^{*}=\sum_{i=1}^{r}\left|E_{j}^{(i)}\right|, \quad$ and $\sigma_{j}=\sqrt{\frac{\sum_{i=1}^{r}\left(E_{j}^{(i)}-\mu_{j}\right)}{r}}$

where $\mu_{j}=\sum_{i=1}^{r} E_{j}^{(i)}$. If $\mu_{j}^{*}$ and/or $\sigma_{j}$ are significantly different from zero, then input $j$ has an important influence on the output. A large index $\sigma_{j}$ indicates either a highly non-linear effect on the output or some interactions between input $j$ and other inputs. These measures can be plotted on a graph where the $x$ - and $y$-axes are the modified means and standard deviations, respectively.

\subsection{Sobol indices}

In a non-linear and non-monotonic model framework, it is possible to estimate the impact of inputs on outputs by using the decomposition of a function $z$ into a sum of elementary functions [22]:

$z\left(X_{1}, \ldots, X_{n}\right)=z_{0}+\sum_{i=0}^{n} z_{i}\left(X_{i}\right)+\sum_{i<j}^{n} z_{i j}\left(X_{i}, X_{j}\right)+\cdots+z_{12 \ldots n}\left(X_{1}, \ldots, X_{n}\right)$

where $z$ is integrable on $\Omega=[0,1]^{n}, z_{0}$ is a constant and other functions have particular characteristics. This decomposition was extended to the sensitivity analysis by Sobol [21], hence, the name Sobol decomposition. One of its contributions was to prove that this decomposition is unique. If the $X_{i}$ are independent random variables, the functional variance decomposition, that is, the functional ANOVA representation, can be obtained from (17):

$\operatorname{Var}[Y]=\sum_{i=0}^{n} V_{i}(Y)+\sum_{i<j}^{n} V_{i j}(Y)+\sum_{i<j<k}^{n} V_{i j k}(Y)+\cdots+V_{12 \ldots n}(Y)$

where $V_{i}(Y)=\operatorname{Var}\left[\mathbb{E}\left(Y \mid X_{i}\right)\right], V_{i j}(Y)=\operatorname{Var}\left[\mathbb{E}\left(Y \mid X_{i} X_{j}\right)\right]-V_{i}(Y)-V_{j}(Y)$, and so on. Accordingly, the Sobol sensitivity indices are defined as follows:

$S_{i}=\frac{\operatorname{Var}\left[\mathbb{E}\left(Y \mid X_{i}\right)\right]}{\operatorname{Var}(Y)}=\frac{V_{i}(Y)}{\operatorname{Var}(Y)}, \quad S_{i j}=\frac{V_{i j}(Y)}{\operatorname{Var}(Y)}, \quad S_{i j k}=\frac{V_{i j k}(Y)}{\operatorname{Var}(Y)}, \ldots$

These coefficients are "variance based importance measures" or Sobol indices. Ranging from 0 to 1 , they are quite easy to interpret, which explains their popularity. For example, index $S_{i j}$ traduces the model sensitivity to the interaction between $X_{i}$ and $X_{j}$. The sum of these indices is equal to 1 . When the input $n$ number increases, the number of Sobol indices grows exponentially (equal to $2^{n-1}$ ). Homma and Saltelli [23] introduced the notion of the total sensitivity index to express the whole effect of an input on the output, thus simplifying their interpretation:

$S_{T i}=S_{i}+\sum_{j \neq i}^{n} S_{i j}+\sum_{j \neq i, k \neq i, j<k}^{n} S_{i j k}+\cdots=\sum_{l \in \# i} S_{l}$

where $\# i$ represents all the indices subsets containing index $i$. Thus, $\sum_{l \in \# i} S_{l}$ is the sum of all the Sobol indices involving $i$. In practice, when $n$ is large, only first order indices and total indices are utilized.

To estimate Sobol indices, we choose to use the Monte-Carlo based method developed by Sobol [21] and Saltelli [24]. The main disadvantage of the Monte-Carlo method is that the number of model evaluations needed to reach $10 \%$ precision is sometimes nearly 10,000 , which means that in cases where the model is computationally time-demanding, this method is not feasible. As proposed in [25], we have chosen to compute these indices from a surrogate model to reduce the computation costs. Note that the computation of Sobol indices can be performed using the FERUM open-source Matlab ${ }^{\mathrm{TM}}$ toolbox [26].

\subsection{Global sensitivity analysis strategy}

Finally, two types of sensitivity analysis methods are addressed in this paper:

- The Morris method: a coarse and rather qualitative method, but it can be performed with limited calls to the model. In this paper, it is used on the complete physics-based model to determine the list of uncertain parameters.

- Sobol sensitivity indices: a fine and quantitative method, but it necessitate a very large number of calls to the model. In this paper, it is used on a surrogate model to hierarchize the parameters and target the uncertainties quantification priorities.

The global sensitivity analysis strategy is divided into the following steps:

1. Determining the initial set of parameters.

2. Performing a coarse quantification of uncertainties. Typically, we can determine the minimum and maximum reasonable values of parameters from expert knowledge such that at this step the PDFs of the parameters are uniform.

3. Performing Morris method from these uniform PDFs. Determining the list of uncertain parameters.

4. Building the surrogate model (c.f. Section 5).

5. Computing Sobol indices from uniform PDFs with uncertain parameters as inputs. Hierarchizing the uncertain parameters and determining the most influent ones.

6. Performing a fine quantification of uncertainties for the most influent uncertain parameters. Estimating the type and parameters of their PDFs.

7. Running uncertainties propagation on the surrogate model with uncertain parameters as inputs. 


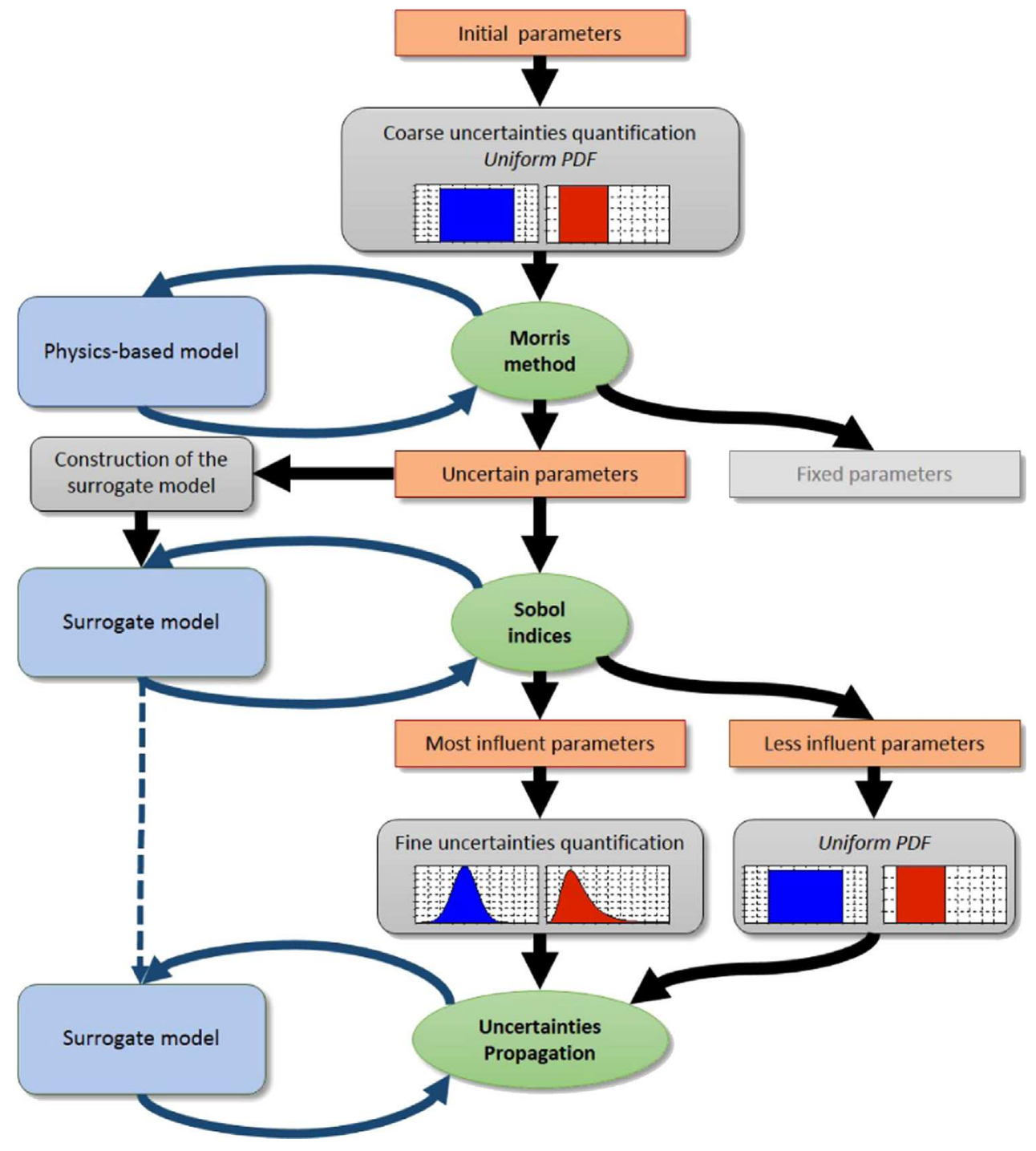

Fig. 5. Uncertainties management and sensitivity analysis.

The global sensitivity analysis scheme proposed in this paper is presented in Fig. 5.

\section{5. kriging surrogate modeling}

In this section, the surrogate modeling is introduced. Its usefulness is twofold: Not only it allows computing a simplified model of the system but it also enables the computation of Sobol indices.

\subsection{Surrogate modeling}

When physics-based models are time-demanding, it is plausible to use surrogate modeling, which is a low cost model of a model in terms of computation time. For example, surrogate modeling has been used to optimize aerospace design [27] because the simulation of the airflow around the wing profiles is highly computationally time-demanding. The construction of a surrogate model is generally composed of the following steps (see Fig. 6):

1. Determination of the variation range of input parameters. In this paper, the variation range is determined by the coarse quantification of uncertainties presented in the previous section.

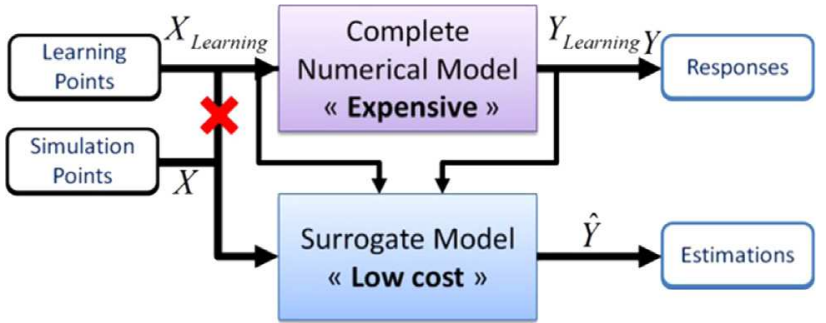

Fig. 6. Principle of surrogate modeling. The low cost model is constructed from $X_{\text {Learning }}$ and $Y_{\text {Learning }}$.

2. Construction of the learning DOE. In the present case, we have chosen to use the Latin hypercube sampling, as introduced in Section 5.2 .

3. Estimation of the surrogate model hyperparameters from a set of learning points. In this work, we have chosen to use kriging, as presented in Section 5.3.

\subsection{Design of experiment}

As shown in Fig. 6, some learning points, also called design sites, are required to build a surrogate model. To optimize the sites 
selection, DOEs are constructed. Even if the DOE to be used depends on the type of surrogate model, the choice is typically made among low discrepancy DOEs. Indeed, this type of DOE allows for an exhaustive search of the range of variations with respect to model parameter space. For example, the Latin hypercube sampling (LHS) method is widely used with kriging to create design sites from multidimensional PDFs of $\mathrm{p}$ variables. The LHS consists of the following steps:

1. Discretization of the $\mathrm{p}$ PDF into $\mathrm{n}$ intervals with equal probability. Intervals are noted as $\mathscr{F}_{1}^{\mathrm{p}}, \ldots, \mathscr{J}_{\mathrm{n}}^{\mathrm{p}}$.

2. Creation of a permutation matrix $\boldsymbol{A} \in \mathbb{N}^{n \times p}$ :

$$
\boldsymbol{A}=\left|\begin{array}{ccc}
\sigma^{1}(1) & \cdots & \sigma^{p}(1) \\
\vdots & \ddots & \vdots \\
\sigma^{1}(n) & \cdots & \sigma^{p}(n)
\end{array}\right|
$$

where $\sigma^{1}, \ldots, \sigma^{\mathrm{p}}$ are permutations of $[1 ; n]$

3. Random sampling according to the different PDFs to construct the DOE:

$\mathbf{D O E}=\left|\begin{array}{ccc}\operatorname{rand}\left(\mathscr{F}_{\sigma^{1}(1)}^{1}\right) & \cdots & \operatorname{rand}\left(\mathscr{F}_{\sigma^{p}(1)}^{p}\right) \\ \vdots & \ddots & \vdots \\ \operatorname{rand}\left(\mathscr{F}_{\sigma^{1}(n)}^{1}\right) & \cdots & \operatorname{rand}\left(\mathscr{F}_{\sigma^{p}(n)}^{p}\right)\end{array}\right|$

where rand is a function drawing randomly a value according to an interval PDF. The main advantage of LHS is that under certain hypotheses not detailed in this paper, it is possible to obtain a DOE with a low discrepancy not only in the global space but also in each single dimension.

\subsection{Kriging}

Kriging, also called a Gaussian process, was initially developed by the mining engineer Daniel Krige for interpolation in geostatistics before being applied to numerical modeling. See [28] for a recent survey. A kriging model can be written as follows:

$\boldsymbol{Y}(\boldsymbol{x})=\boldsymbol{f}^{T}(\boldsymbol{x}) \boldsymbol{b}+Z(\boldsymbol{x})$

where $x$ is a point in a $d$-dimensional input space, $f^{T}(x) b$ is a regression model and $Z$ is a Gaussian process of mean zero and covariance $\sigma^{2} \mathscr{R}\left(\theta, x_{i}, x_{j}\right)$ with $\mathscr{R}$ an assumed correlation function between outputs and inputs such that:

$\mathscr{R}\left(\boldsymbol{\theta}, \boldsymbol{x}_{\boldsymbol{i}}, \boldsymbol{x}_{\mathbf{j}}\right)=\prod_{k=1}^{d} \mathscr{R}_{k}\left(\theta_{k}, x_{i k}, x_{j k}\right)$

The kriging model hyperparameters $\theta, b$ and $\sigma^{2}$ are generally computed by maximum likelihood estimation. Some examples of correlation functions are given in Table 1 . These functions imply that $Y\left(x_{i}\right)$ and $Y\left(x_{j}\right)$ are more correlated as their input locations $x_{i}$ and $x_{j}$ are closer. While the choice of the correlation function is of paramount importance because it determines the quality of the

Table 1

Different types of correlation models for kriging.

\begin{tabular}{ll}
\hline Correlation type & $\mathscr{R}_{\boldsymbol{k}}\left(\boldsymbol{\theta}_{\boldsymbol{k}}, \boldsymbol{x}_{\boldsymbol{i} \boldsymbol{k}}, \boldsymbol{x}_{\boldsymbol{j} \boldsymbol{k}}\right)$ \\
\hline Exponential & $\exp \left(-\theta_{k}\left|x_{j k}-x_{i k}\right|\right)$ \\
Gaussian & $\exp \left(-\theta_{j}\left|x_{j k}-x_{i k}\right|^{2}\right)$ \\
Exponential - Gaussian & $\exp \left(-\theta_{j}\left|x_{j k}-x_{i k}\right|^{\theta_{n+1}}\right), \quad 0<\theta_{n+1} \leq 2$ \\
Linear & $\max \left\{0,1-\theta_{k}\left|x_{j k}-x_{i k}\right|\right\}$ \\
Spherical & $1-1.5 \xi_{j}+0.5 \xi_{j}^{3}, \quad \xi_{j}=\min \left\{1, \theta_{k}\left|x_{j k}-x_{i k}\right|\right\}$ \\
Cubic & $1-3 \xi_{j}^{2}+2 \xi_{j}^{3}, \quad \xi_{j}=\min \left\{1, \theta_{k}\left|x_{j k}-x_{i k}\right|\right\}$ \\
\hline
\end{tabular}

kriging model estimations, it depends on the characteristics of the model. For example, a Gaussian correlation suits generally well the linear models, whereas an exponential correlation is more adapted to non-linear models.

From n observations $Y=\left(y_{1}, \ldots, y_{n}\right)^{T}$ corresponding to design sites $X=\left(x_{1}, \ldots, x_{n}\right)^{T}$, kriging uses best linear unbiased predictor (BLUP) criterion to minimize the mean squared error of the predictor. For a point $x_{n+1}$, the kriging predictor is

$\hat{\boldsymbol{Y}}\left(\boldsymbol{x}_{\boldsymbol{n}+1}\right)=\boldsymbol{f}^{T}\left(\boldsymbol{x}_{\boldsymbol{n}+1}\right) \boldsymbol{b}+\boldsymbol{r}\left(\boldsymbol{x}_{\boldsymbol{n}+1}\right)^{T} \boldsymbol{R}^{-1}(\boldsymbol{Y}-\boldsymbol{F} \boldsymbol{b})$

where $b$ is the matrix of the regression coefficients, $R$ is the correlation matrix, $F=\left(f\left(x_{1}\right), \ldots, f\left(x_{n}\right)\right)^{T}$ and $r$ is the correlation function between $x_{n+1}$ and design sites such that:

$\boldsymbol{r}\left(\boldsymbol{x}_{\boldsymbol{n}+1}\right)=\left[\mathscr{R}\left(\boldsymbol{\theta}, \boldsymbol{x}_{1}, \boldsymbol{x}_{\boldsymbol{n}+1}\right) \cdots \mathscr{R}\left(\boldsymbol{\theta}, \boldsymbol{x}_{\boldsymbol{n}}, \boldsymbol{x}_{\boldsymbol{n}+1}\right)\right]^{T}$

It is determined that if $x_{n+1}$ coincides with a design site, the predictor equals the observation. Thus, the kriging predictor is an exact interpolator. Thus, it is possible to calculate the variance of the prediction $\Sigma^{2}$ at any point $x$ :

$\left.\Sigma^{2}(\boldsymbol{x})=\sigma^{2} \quad 1-\boldsymbol{r}^{T} \boldsymbol{R}^{-1} \boldsymbol{r}+\frac{\left(1-1^{T} \boldsymbol{R}^{-1} \boldsymbol{r}\right)^{2}}{1^{T} \boldsymbol{R}^{-1} 1}\right)$

Finally, the kriging predictor has three main advantages: it is a BLUP, it is an exact interpolator on design sites and it is capable of estimating its own prediction variance. A kriging toolbox available for the software Matlab ${ }^{\mathrm{TM}}$ is introduced in [29]. This toolbox proposes an algorithm for the estimation of the kriging hyperparameters that is used in the following application.

\section{Application system}

\subsection{System presentation}

For the higher purpose of monitoring the whole fuel system of the aircraft engine, it is necessary to monitor all its critical subsystems, for example, the hydromechanical loops as explained in [30]. In this paper, the studied system is a pumping unit of an aircraft engine fuel system [31]. This system is composed of a centrifugal low pressure pump and a gear high pressure pump. The pumping unit is located in the fuel system, as shown in Fig. 7, and interacts with the following equipments:

- BSV: Burning stage valve to switch between 1 and 2 injector lines

- TBV: Transient bleed valve to produce a discharge

- HPSOV: High pressure shut off valve to maintain the pressurization of the system $\Delta P=P_{H P}-P_{L P}$

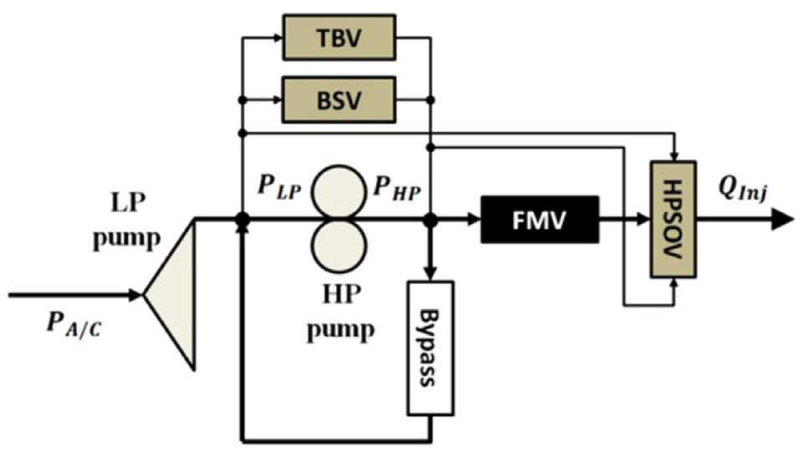

Fig. 7. Aircraft fuel system scheme with $P_{A / C}$ aircraft pressure supply, $P_{L P}$ and $P_{H P}$, respectively, low and high pressures of the system and $Q_{\text {Inj }}$ injection flow. 
Table 2

Degradation modes and degradation parameters.

\begin{tabular}{|c|c|c|}
\hline Degradation mode & Modeling method & Degradation parameter \\
\hline 1. HP pump internal leakage & Orifice between pump outlet and pump inlet & Orifice diameter: $\boldsymbol{D}_{\boldsymbol{I L k g}}$ \\
\hline 2. HP pump external leakage & Orifice between pump outlet and ambient pressure tank & Orifice diameter: $\boldsymbol{D}_{\mathbf{E L k g}}$ \\
\hline 3. BSV striction increase & Increase of striction coefficient & Striction coefficient: $\boldsymbol{S} \boldsymbol{t}_{\boldsymbol{B S V}}$ \\
\hline 4. BSV viscous friction increase & Increase of viscous coefficient & Viscous coefficient: $\boldsymbol{V}_{\boldsymbol{B S V}}$ \\
\hline 5. BSV leakage & Orifice between the two chambers of the valve & Orifice diameter: $\boldsymbol{D}_{\mathbf{B S V}}$ \\
\hline 6. Bypass striction increase & Increase of striction coefficient & Striction coefficient: $\boldsymbol{S} \boldsymbol{t}_{\boldsymbol{B} y \boldsymbol{p}}$ \\
\hline 7. Bypass viscous friction increase & Increase of viscous coefficient & Viscous coefficient: $\boldsymbol{V i}_{\boldsymbol{B y p}}$ \\
\hline 8. Bypass leakage & Orifice between the two chambers of the valve & Orifice diameter: $\boldsymbol{D}_{\boldsymbol{B y p}}$ \\
\hline 9. HPSOV striction increase & Increase of striction coefficient & Striction coefficient: $\boldsymbol{S} \boldsymbol{t}_{\text {HPSOV }}$ \\
\hline 10. HPSOV viscous friction increase & Increase of viscous coefficient & Viscous coefficient: $\boldsymbol{V} \boldsymbol{i}_{\text {HPSOV }}$ \\
\hline 11. HPSOV leakage & Orifice between the two chambers of the valve & Orifice diameter: $\boldsymbol{D}_{\text {HPSOV }}$ \\
\hline 12. TBV striction increase & Increase of striction coefficient & Striction coefficient: $\boldsymbol{S} \boldsymbol{t}_{\boldsymbol{T B V}}$ \\
\hline 13. TBV viscous friction increase & Increase of viscous coefficient & Viscous coefficient: $\boldsymbol{V} \boldsymbol{i}_{\boldsymbol{T B V}}$ \\
\hline 14. TBV leakage & Orifice between the two chambers of the valve & Orifice diameter: $\boldsymbol{D}_{T B V}$ \\
\hline
\end{tabular}

- FMV: Fuel metering valve to regulate the amount of injected fuel

- Bypass: Valve to redirect the excess of pumped fuel into the pump inlet

\subsection{System analysis}

\subsubsection{Failure modes}

The failure modes of the system have been determined by experience feedback and verified by expertise. They are listed in Table 2 with their associated modeling method and degradation parameters. Among these degradation modes, two have been identified as critical: the HP pump internal and external leakage. Indeed, a failure modes, effects, and criticality analysis (FMECA) [32] performed on the system from expert knowledge and experience feedback revealed that both these degradation modes can lead to the highly critical event defined in the system's specifications: "the outlet flow at $10 \%$ of the maximal rotation speed of the pump is inferior to $Q_{m}$ ", where $Q_{m}$ is the minimal theoretical value of the flow that ensures both the engine on-ground start and in-flight restart capabilities. On the contrary, as the other degradation modes do not lead to critical events, they are considered as marginal. However, it was necessary to take the other modes into account for the evaluation of identification potential. Indeed, they can influence the values of the HIs, and they can be confused with critical degradation modes, which can lead to false prognostics and unnecessary maintenance operations.

\subsubsection{Health indicators}

The selection of HIs can be a complex task in cases were the number, location and characteristics of sensors are imposed. Indeed, if it is not possible to organize sensors to set up a direct monitoring of the system, the solution is to find indirect ways to follow the evolution of degradation modes. In the present case, the best solution to monitor degradation modes is to monitor their common effect, i.e., when the outlet flow at $10 \%$ of the maximal rotation speed of the pump is inferior to $Q_{m}$. However, if this value is available, which is not the case in real life service, it is due to a lack of sensors. Actually, the only values available online are:

- $\omega$ : rotation speed of the pump (via a reduction ratio from the high pressure core rotation speed)

- OSOV: Boolean equal to 1 if the HPSOV is open and to 0 if it is closed. It is computed from a proximeter.

- $O_{T B V}$ : Boolean equal to 1 if the TBV is open and to 0 if it is closed. It is computed from a proximeter.

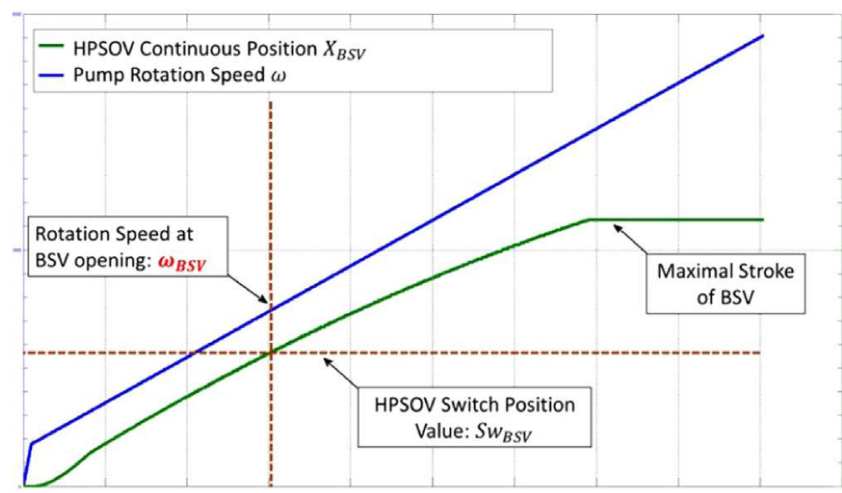

Fig. 8. Example of HI extraction: the case of $w B S V$.

- $O_{B S V}$ : Boolean equal to 1 if the BSV is open and to 0 if it is closed. It is computed from a proximeter.

- $X_{F M V}$ : Value of the FMV position, equal to 0 during the start sequence.

To define a HI to monitor indirectly the outlet flow of the pump, the idea is to find variables that are images of the hydraulic power gradient over the starting sequence. It appears that the three valves, HPSOV, BSV and TBV, are in a close position at the beginning of the start sequence $(t=0)$, but their command requires them to be open. However, as the system is not pressurized at $t=0$, the valves cannot move and their opening occurs only when the hydraulic power has reached a minimal value. Thus, considering the evolution of the necessary rotation speed to open the different valve is a good way to assess the health status of the pump. Accordingly, we define the following HIs, which were validated by expert knowledge:

- $\omega_{\text {SOV }}$ : Rotation speed of the pump at the HPSOV opening, i.e., $\omega_{S O V}=\min \left\{\omega / O_{S O V}=1\right\}$.

- $\omega_{B S V}$ : Rotation speed of the pump at the BSV opening, i.e., $\omega_{B S V}=\min \left\{\omega / O_{B S V}=1\right\}$.

- $\omega_{T B V}$ : Rotation speed of the pump at the TBV opening, i.e., $\omega_{T B V}=\min \left\{\omega / O_{T B V}=1\right\}$.

The a priori advantage of these HIs is that in real life service, the values are easily retrievable as only the value of the rotation speed when Booleans indicating the positions of the valves switch from 0 to 1 needs to be stored. In simulation, we do not have the Boolean values, but we do have the continuous value of the valve's spool position. Thus, to retrieve the HIs, we need the values of the 
Table 3

Epistemic and context parameters.

\begin{tabular}{|c|c|c|c|c|c|}
\hline Parameter & Type & Parameter & Type & Parameter & Type \\
\hline Fuel temperature: $\boldsymbol{T}_{\text {fuel }}$ & Ctx & Bypass feedback stiffness: $\boldsymbol{K}_{\boldsymbol{B} \boldsymbol{y} \boldsymbol{p}}$ & Epi & TBV rod diameter: $\boldsymbol{D} \boldsymbol{R}_{\boldsymbol{T B V}}$ & Epi \\
\hline Aircraft supply pressure: $\boldsymbol{P}_{\boldsymbol{A} / \boldsymbol{C}}$ & Ctx & Bypass head diameter: $\mathbf{D H}_{\mathbf{B y p}}$ & Epi & TBV opening pressure: $\boldsymbol{P o p}_{T B V}$ & Epi \\
\hline Injection pressure: $\boldsymbol{P}_{\boldsymbol{i n j}}$ & Ctx & Bypass rod diameter: $\boldsymbol{D} \boldsymbol{R}_{\boldsymbol{B y p}}$ & Epi & BSV mass: $\boldsymbol{M}_{\boldsymbol{B S V}}$ & Epi \\
\hline Second order coefficient of $N 2(t): \boldsymbol{A}_{\boldsymbol{N} 2}$ & Ctx & Bypass strength calibration: $\boldsymbol{C}_{\boldsymbol{B} \boldsymbol{y p}}$ & Epi & BSV feedback stiffness: $\boldsymbol{K}_{\boldsymbol{B S V}}$ & Epi \\
\hline First order coefficient of $N 2(t): \boldsymbol{B}_{N 2}$ & Ctx & HPSOV mass: $\boldsymbol{M}_{\text {HPSOV }}$ & Epi & BSV head diameter: $\boldsymbol{D H}_{\boldsymbol{B S V}}$ & Epi \\
\hline Opening stroke for BSV: $\boldsymbol{S} \boldsymbol{w}_{\boldsymbol{B} \boldsymbol{}}$ & Epi & HPSOV feedback stiffness: $\boldsymbol{K}_{\text {HPSOV }}$ & Epi & BSV rod diameter: $\boldsymbol{D} \boldsymbol{R}_{\boldsymbol{B S V}}$ & Epi \\
\hline Opening stroke for TBV: $\boldsymbol{S} \boldsymbol{w}_{T B V}$ & Epi & HPSOV head diameter: $\boldsymbol{D H}_{\text {HPSOV }}$ & Epi & BSV strength calibration: $\boldsymbol{C}_{\boldsymbol{B S V}}$ & Epi \\
\hline Opening stroke for HPSOV: $\boldsymbol{S} \boldsymbol{w}_{\text {HPSOV }}$ & Epi & HPSOV strength calibration: $\boldsymbol{C}_{H P S O V}$ & Epi & BSV servo gain: $\boldsymbol{G}_{S V B S V}$ & Epi \\
\hline HP pump displacement: Dis & Epi & TBV mass: $\boldsymbol{M}_{T B V}$ & Epi & BSV servo damping: $\boldsymbol{D}_{\text {SVBSV }}$ & Epi \\
\hline Bypass mass: $\boldsymbol{M}_{\boldsymbol{B} y \boldsymbol{p}}$ & Epi & TBV head diameter: $\boldsymbol{D H}_{\mathbf{T B V}}$ & Epi & & \\
\hline
\end{tabular}

switch positions $S w_{S O V}, S w_{B S V}$ and $S w_{T B V}$. These values can be found in the equipment's specifications. Finally, the HIs from the model are computed as follows:

$\left\{\begin{array}{c}\omega_{S O V}=\min \left\{\omega / X_{S O V}>S w_{S O V}\right\} \\ \omega_{B S V}=\min \left\{\omega / X_{B S V}>S w_{B S V}\right\} \\ \omega_{T B V}=\min \left\{\omega / X_{T B V}>S w_{T B V}\right\}\end{array}\right.$

where $X_{S O V}, X_{B S V}$, and $X_{T B V}$ are, respectively, the continuous positions of HPSOV, BSV and TBV.

The model is run for simulations of the engine starting sequence, i.e., for the seven first seconds of a classical flight scheme. The input of the system is the rotation speed of the high pressure turbine $\boldsymbol{N} 2(\boldsymbol{t})$. This rotation speed is a second degree polynomial function of time such that

$\mathrm{N} 2(t)=A_{N 2} \times t^{2}+B_{N 2} \times t$

Fig. 8 presents how $\boldsymbol{w B S V}$ is recorded from the signal of its position considering that the valve opening is confirmed at $50 \%$ of the stroke. As one simulation of the model is approximately 2 min long, it is too expensive to run a Monte-Carlo algorithm for uncertainties propagation. In the next section, the use of kriging to decrease the computational costs is discussed.

\subsection{System modeling}

\subsubsection{Model parameters}

The modeled system is composed of 43 parameters, including 5 context parameters, 24 epistemic parameters and 14 degradation parameters. The list of context and epistemic parameters is provided in Table 3 . The list of degradation parameters is presented in Table 2 .

\section{Results}

\subsection{Uncertain parameters}

\subsubsection{Coarse quantification of uncertainties}

All the parameters defined in Table 3 are subject to uncertainties of a random or systematic type, respectively, for context and random parameters. In a first time, we suppose that the distributions of parameters are uniform. The minimum and maximum values are evaluated via the analysis of experience feedback on other engines, whereas systematic uncertainties are determined through the analysis of equipment specifications and, particularly, confidence intervals given by manufacturers. For the degradation parameters, we also use uniform laws with the minimum value equal to the nominal value and the maximum value determined from expert knowledge. The results of these coarse uncertainties quantifications are given in Table 4.
In Table 4, the parameters are assumed to be independent. This assumption was verified either by the computation of the correlation coefficient or by the analysis of scatter plots for uncertainties that can be measured on similar systems, namely, $T_{f u e l}, P_{A / C}, P_{i n j}$, $A_{N 2}$, and $B_{N 2}$. For other parameters, this hypothesis was not verified but strongly presumed via physical consideration from expert knowledge. Note that it can be verified from simulations after the kriging model is constructed.

\subsubsection{Morris method}

The Morris method is performed for a model with a parameters space dimension equal to 43 . We chose a number of repetitions equal to 5 so the model is called 215 times, which is equivalent to approximately $7 \mathrm{~h}$ of computation. The results of the Morris method corresponding to each $\mathrm{HI}$ are presented in Fig. 9:

Using Fig. 9, we can hierarchize the parameters with respect to their influence on each of the outputs. This influence is proportional to the distance between the points and the origin. From the results of this sorting, it appear that 23 parameters have negligible influence on the three HIs and that the 20 remaining parameters have significant influence Hence, the former are identified as fixed parameters and the latter as uncertain parameters. The Morris method also allows for the qualitative evaluation of the supposed effects of each uncertain parameter on the three outputs. Table 5 shows an example of effects evaluation for HI $w B S V$, revealing that only 7 out of 14 degradation modes actually have influence on the HIs. We notice that the critical degradation modes $\left(\boldsymbol{D}_{\mathbf{I L k g}}\right.$ and $\left.\boldsymbol{D}_{\boldsymbol{I L k g}}\right)$ are expected to have a strong effect on $w B S V$, which is a good point for detection purposes.

\subsection{Influent parameters}

\subsubsection{Kriging model}

In this application, the kriging model is a function aimed at estimating WBSV, $\boldsymbol{W T B V}$ and $\boldsymbol{w H P S O V}$. We define the inputs of the kriging model as the uncertain parameters determined in the previous subsection. The learning sites are constructed via a LHS of size 400 and uniform distributions to ensure a good space filling. The kriging model is then built using a first degree polynomial regression and an exponential correlation. The estimation of kriging hyperparameters is performed using the DACE algorithm [33].

\subsubsection{Sobol indices}

The Sobol indices of the 20 inputs presented in Table 5 are computed using the Monte-Carlo method performed on the kriging model constructed in the previous subsection. For this application, both first order and total indices are computed from the MCS for a number of iterations $q=100,000$. The results are presented in Fig. 10 for each $\mathrm{HI}$ 
Table 4

Coarse uncertainties quantification results.

\begin{tabular}{|c|c|c|c|c|c|}
\hline Param & Pdf & Param & Pdf & Param & Pdf \\
\hline$T_{\text {fuel }}$ & $\mathbf{U}(-40,30)$ & $K_{H P S O V}$ & $\mathbf{U}(0.99,1.01)$ & $D_{E L k g}$ & $\mathbf{U}(0,1.5)$ \\
\hline$P_{A / C}$ & $\mathbf{U}(1.9,2.1)$ & DH HPSOV & $\mathbf{U}(24,26)$ & $S t_{B S V}$ & $\mathbf{U}(0,100)$ \\
\hline$P_{i n j}$ & $\mathbf{U}(0.95,1.05)$ & $C_{H P S O V}$ & $\mathbf{U}(19,21)$ & $V i_{B S V}$ & $\mathbf{U}(50,2000)$ \\
\hline$A_{N 2}$ & $\mathbf{U}(1.7,2.3)$ & $M_{T B V}$ & $\mathbf{U}(0.2,0.22)$ & $D_{B S V}$ & $\mathbf{U}(0,0.5)$ \\
\hline $\boldsymbol{B}_{N 2}$ & $\mathbf{U}(0.96,1.04)$ & $D H_{T B V}$ & $\mathbf{U}(42.22,46.22)$ & $S t_{\text {Byp }}$ & $\mathbf{U}(0,100)$ \\
\hline$S w_{B S V}$ & $\mathbf{U}\left(2.3 \boldsymbol{e}^{-03}, 2.7 \boldsymbol{e}^{-03}\right)$ & $D R_{T B V}$ & $\mathbf{U}(18,20)$ & $V i_{\text {Byp }}$ & $\mathbf{U}(50,2000)$ \\
\hline$S w_{T B V}$ & $\mathbf{U}\left(1.2 \boldsymbol{e}^{-02}, 1.3 \boldsymbol{e}^{-02}\right)$ & $\operatorname{Pop}_{T B V}$ & $\mathbf{U}(7,15)$ & $D_{\text {Byp }}$ & $\mathbf{U}(0,0.5)$ \\
\hline Sw $w_{\text {HPSOV }}$ & $\mathbf{U}\left(0.9,1.1 \boldsymbol{e}^{-03}\right)$ & $M_{B S V}$ & $\mathbf{U}(0.38,0.40)$ & $S t_{\text {HPSOV }}$ & $\mathbf{U}(0,100)$ \\
\hline Dis & $\mathbf{U}\left(2.3 \boldsymbol{e}^{-05}, 2.4 \boldsymbol{e}^{-05}\right)$ & $K_{B S V}$ & $\mathbf{U}(0.99,1.01)$ & $V i_{H P S O V}$ & $\mathbf{U}(0,2000)$ \\
\hline$M_{B y p}$ & $\mathbf{U}(0.18,0.22)$ & $D H_{B S V}$ & $\mathbf{U}(28.87,30.87)$ & $D_{\text {HPSOV }}$ & $\mathbf{U}(0,0.5)$ \\
\hline$K_{\text {Byp }}$ & $\mathbf{U}(0.99,1.01)$ & $D R_{B S V}$ & $\mathbf{U}(5.29,7.29)$ & $S t_{T B V}$ & $\mathbf{U}(0,100)$ \\
\hline$D H_{B y p}$ & $\mathbf{U}(29,31)$ & $C_{B S V}$ & $\mathbf{U}(-2,2)$ & $V i_{T B V}$ & $\mathbf{U}(0,2000)$ \\
\hline$D R_{\text {Byp }}$ & $\mathbf{U}(23,25)$ & $G_{S V B S V}$ & $\mathbf{U}(0.067,0.267)$ & $D_{T B V}$ & $\mathbf{U}(0,0.5)$ \\
\hline$C_{B y p}$ & $\mathbf{U}(-7,-3)$ & $D_{S V B S V}$ & $\mathbf{U}(1.6,2)$ & & \\
\hline$M_{\text {HPSOV }}$ & $\mathbf{U}(0.29,0.31)$ & $D_{\text {ILkg }}$ & $\mathbf{U}(0,1.5)$ & & \\
\hline
\end{tabular}
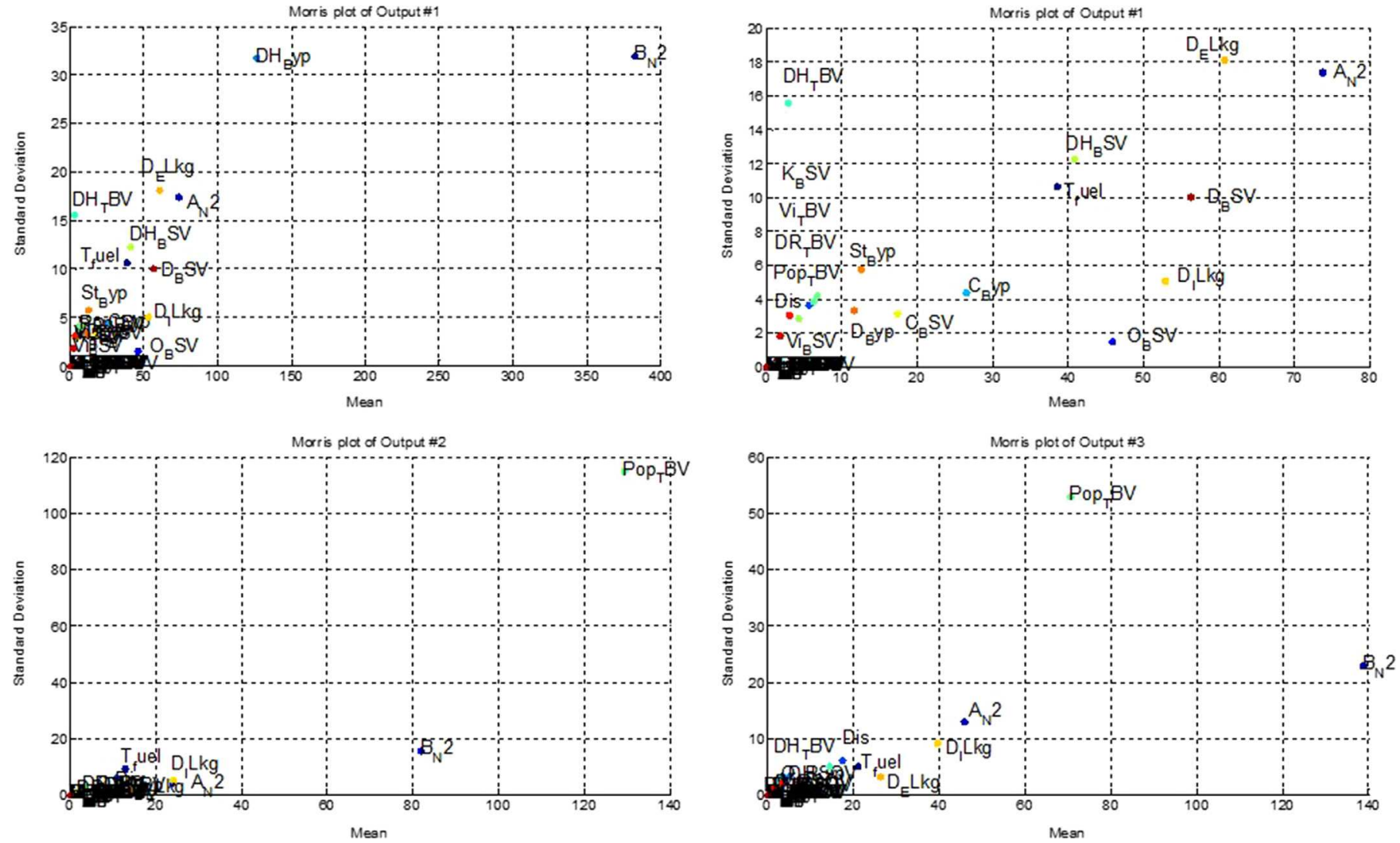

Fig. 9. Morris plot for $w B S V$ (top left), zoomed $w B S V$ (top right), $w T B V$ (bottom left) and $w H P S O V$ (bottom right).

Table 5

List of uncertain parameters.

\begin{tabular}{|c|c|c|c|c|c|c|c|}
\hline Param & Effect & Param & Pdf & Param & Pdf & Param & Pdf \\
\hline 1. $T_{\text {fuel }}$ & Weak non-linear & 6. DH $H_{\text {Byp }}$ & Strong non-linear & 11. $K_{B S V}$ & Weak linear & 16. $S t_{B y p}$ & Weak non-linear \\
\hline 2. $A_{N 2}$ & Strong linear & 7. $C_{B y p}$ & Weak linear & 12. $\mathrm{DH}_{B S V}$ & Average non-linear & 17. $D_{\text {Byp }}$ & Very weak non-linear \\
\hline 3. $B_{N 2}$ & Very strong linear & 8. $\mathrm{DH}_{T B V}$ & Strong non-linear & 13. $C_{B S V}$ & Weak linear & 18. $V i_{T B V}$ & Weak non-linear \\
\hline 4. $S w_{B S V}$ & Average linear & 9. $D R_{T B V}$ & Weak non-linear & 14. $D_{\text {ILkg }}$ & Very strong non-linear & 19. $V i_{B S V}$ & Very weak non-linear \\
\hline 5. Dis & Weak non-linear & 10. Pop $_{T B V}$ & Very strong non-linear & 15. DELkg & Very strong non-linear & 20. $D_{B S V}$ & Average non-linear \\
\hline
\end{tabular}



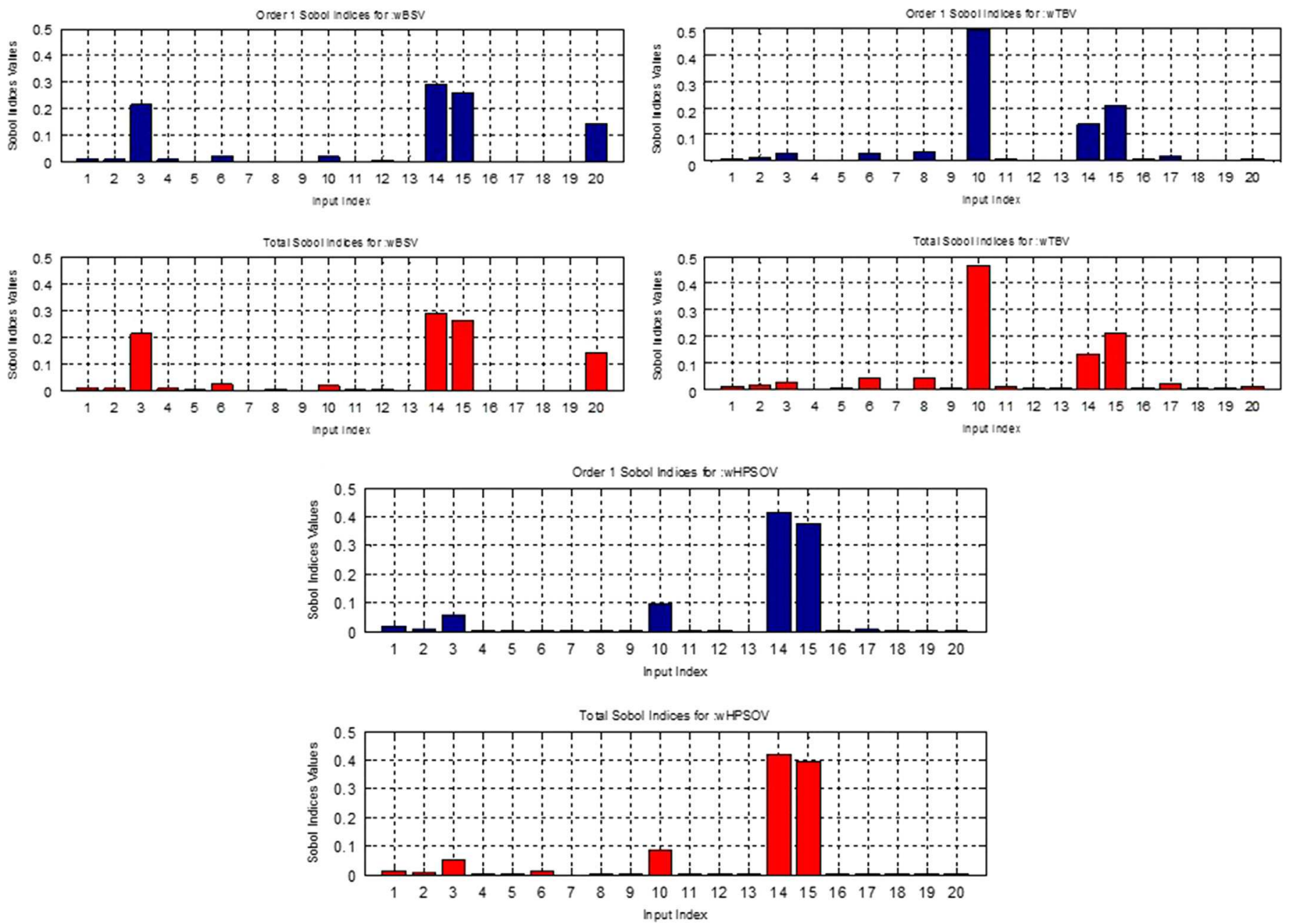

Fig. 10. First order and total Sobol indices for each HI: $w B S V$ (top left), wTBV (top right) and $w H P S O V$ (bottom).

The computation of Sobol indices indicates that the results are not completely correlated with those obtained from the Morris method. Indeed, the most influent parameters for wBSV are $\boldsymbol{D}_{\text {ILkg }}$ and $\boldsymbol{D}_{\text {ELkg }}$ according to Sobol indices, whereas according to the Morris method, the most influent parameters are $\boldsymbol{B}_{\mathbf{N} 2}$ and $\mathbf{D} \boldsymbol{H}_{\boldsymbol{B} y \boldsymbol{p}}$. This difference justifies that both methods should be used. Additionally, the first order indices and the total indices are very close, which indicates that there are few or no correlations between inputs. This observation justifies a posteriori of the independency assumption between parameters. Thus, it is possible to hierarchically sort the most influent parameters for each $\mathrm{HI}$ :

- For $\boldsymbol{w B S V}$, the most influent parameters are, in decreasing order, $\boldsymbol{D}_{\text {ILkg }}, \boldsymbol{D}_{\text {ELkg }}, \boldsymbol{B}_{N 2}$ and $\boldsymbol{D}_{\text {BSV }}$

- For $\boldsymbol{w B S V}$, the most influent parameters are, in decreasing order, $\boldsymbol{P o p}_{\text {TBV }}, \boldsymbol{D}_{\text {ELkg }}$ and $\boldsymbol{D}_{\text {ILkg }}$

- For $\boldsymbol{w B S V}$, the most influent parameters are, in decreasing order, $\boldsymbol{D}_{\text {ILkg }}, \boldsymbol{D}_{\text {ELkg }}, \boldsymbol{P o p}_{\text {TBV }}$ and $\boldsymbol{B}_{\boldsymbol{N} 2}$

\subsection{Uncertainties propagation}

\subsubsection{Fine uncertainties quantification}

As presented in the previous section, the computation of Sobol indices reveal that 5 uncertain parameters are more influent than the others: $\boldsymbol{D}_{\text {ILkg }}, \boldsymbol{D}_{\text {EUkg }}, \boldsymbol{P o p}_{\mathrm{TBV}}, \boldsymbol{B}_{N 2}$ and $\boldsymbol{D}_{\mathrm{BSV}}$. It is notable that both parameters relative to critical degradation modes belong to this list.
Table 6

List of the most influent uncertain parameters with fine uncertainties quantification results.

\begin{tabular}{llll}
\hline Parameter & Pdf & Parameter & Pdf \\
\hline $\boldsymbol{B}_{\mathbf{N} 2}$ & $\mathbf{N}(1,0.01)$ & $\boldsymbol{D}_{\text {ELkg }}$ & $\mathbf{U}(0,1.7)$ \\
Pop $_{\text {TBV }}$ & $\mathbf{N}(11,1.67)$ & $\boldsymbol{D}_{\text {BSV }}$ & $\mathbf{U}(0,0.33)$ \\
$\boldsymbol{D}_{\text {ILkg }}$ & $\mathbf{U}(0,2.1)$ & & \\
\hline
\end{tabular}

We then perform a complete finer uncertainties quantification for these influent parameters and find that $\boldsymbol{B}_{\boldsymbol{N} 2}$ and $\boldsymbol{P o p}_{\mathbf{T B V}}$ were actually normal distribution and the maximum value of degradation parameters was not good. The results are presented in Table 6.

\subsubsection{Uncertainties propagation}

At this point, we have precise PDFs for influent parameters and uniform PDFs for less influent uncertain parameters. Hence, we can perform the uncertainties propagation via Monte-Carlo simulations run on the kriging model, as defined in Section 7.2.1. This propagation is repeated for each triplet (HI $i$, Degradation Mode $j$, Magnitude $\boldsymbol{\omega}$ ) with linearly growing magnitudes. Some of the results are presented in Fig. 11 for the three HIs for degradation mode 1 of growing magnitudes ranging from 0 to 1.5 . Only the individual model case is considered at this time. 

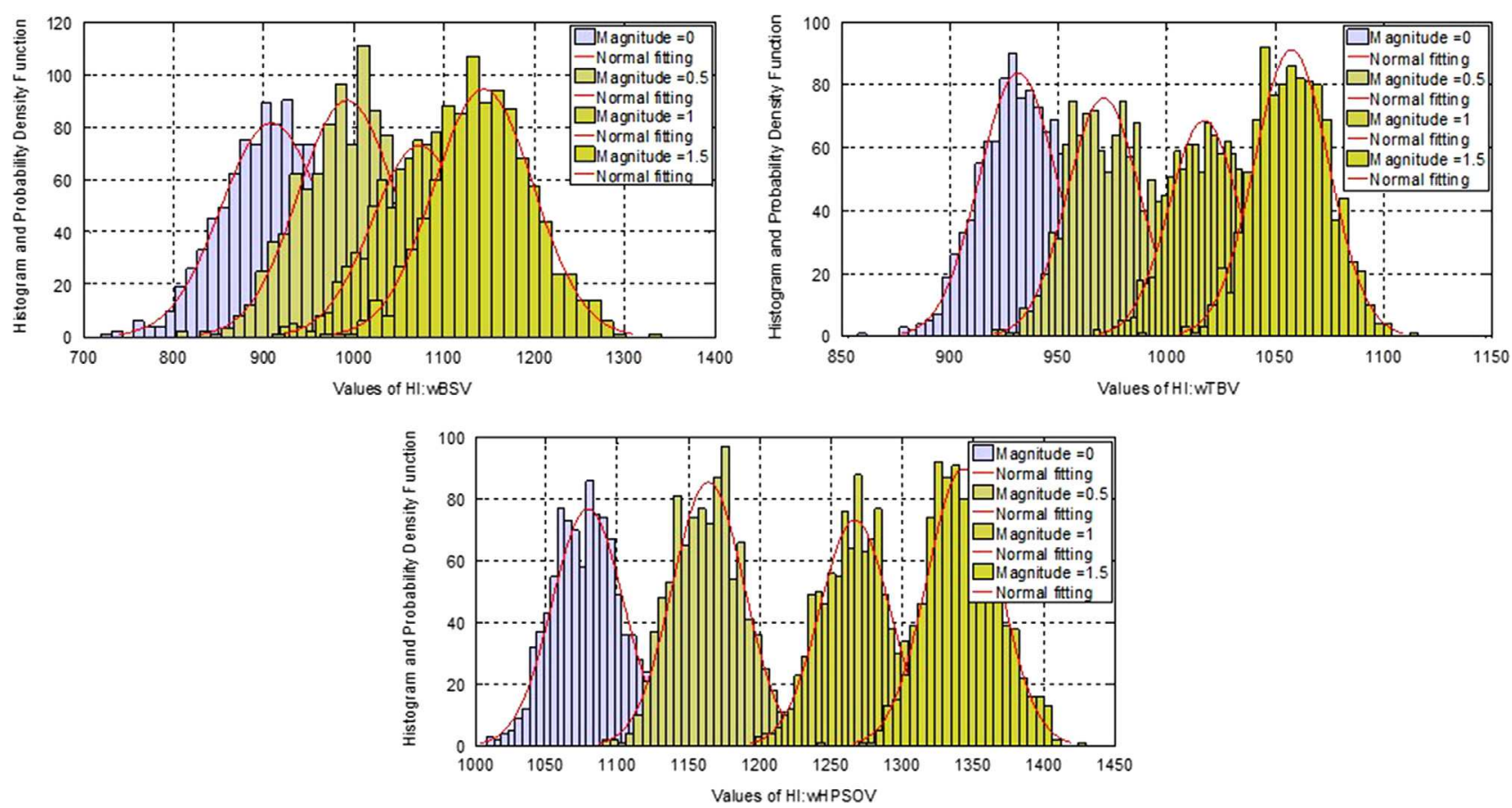

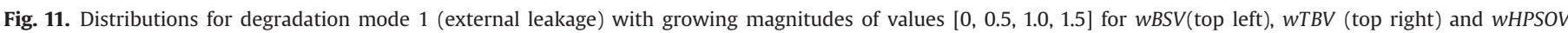
(bottom).

\section{Table 7}

Detection NKPIs for fleet model case.

\begin{tabular}{|c|c|c|c|c|c|c|c|c|c|c|c|c|c|c|c|}
\hline $\begin{array}{c}G D x \\
\text { Fleet }\end{array}$ & $D_{B S V}$ & $D_{B y p}$ & $D_{E L k g}$ & $D_{I L k g}$ & $S t_{B y p}$ & $V i_{B S V}$ & $V i_{T B V}$ & $\begin{array}{c}C D x \\
\text { Indiv }\end{array}$ & $D_{B S V}$ & $D_{\text {Byp }}$ & $D_{E L k g}$ & $D_{I L k g}$ & $S t_{B y p}$ & $V i_{B S V}$ & $V i_{T B V}$ \\
\hline$w B S V$ & 1 & 0.08 & 0.99 & 0.99 & 0.12 & 0.07 & 0 & $w B S V$ & 1 & 0 & 1 & 1 & 0 & 0 & 0 \\
\hline$w T B V$ & 0.1 & 0.37 & 0.94 & 0.83 & 0.39 & 0.07 & 0.01 & $w T B V$ & 0 & 0 & 1 & 0 & 0 & 0 & 0 \\
\hline wHPSOV & 0.45 & 0.04 & 1 & 1 & 0.47 & 0.23 & 0.13 & wHPSOV & 0 & 0 & 1 & 1 & 0 & 0 & 0 \\
\hline
\end{tabular}

Table 8

Detection NKPIs for individual model case.

\begin{tabular}{|c|c|c|c|c|c|c|c|c|c|c|c|c|c|c|c|}
\hline $\begin{array}{c}G D x \\
\text { Fleet } \\
\end{array}$ & $D_{B S V}$ & $D_{B y p}$ & $D_{E L k g}$ & $D_{I L k g}$ & $S t_{B y p}$ & $V i_{B S V}$ & $V i_{T B V}$ & $\begin{array}{c}C D x \\
\text { Indiv } \\
\end{array}$ & $D_{B S V}$ & $D_{\text {Byp }}$ & $D_{E L k g}$ & $D_{I L k g}$ & $S t_{B y p}$ & $V i_{B S V}$ & $V i_{T B V}$ \\
\hline$w B S V$ & 1 & 0.16 & 1 & 1 & 0.12 & 0.09 & 0.03 & $w B S V$ & 1 & 0 & 1 & 1 & 0 & 0 & 0 \\
\hline$w T B V$ & 0.08 & 1 & 1 & 1 & 0.84 & 0.03 & 0.35 & $w T B V$ & 0 & 1 & 1 & 1 & 0 & 0 & 0 \\
\hline wHPSOV & 0.62 & 0.22 & 1 & 1 & 0.64 & 0.33 & 0.14 & wHPSOV & 0 & 0 & 1 & 1 & 0 & 0 & 0 \\
\hline
\end{tabular}

Table 9

Identification NKPIs for fleet and individual model cases.

\begin{tabular}{|c|c|c|c|c|c|c|c|c|c|c|c|c|c|c|c|}
\hline $\begin{array}{c}\text { CIx } \\
\text { Fleet }\end{array}$ & $D_{B S V}$ & $D_{B y p}$ & $D_{E L k g}$ & $D_{\text {ILkg }}$ & $S t_{B y p}$ & $V i_{B S V}$ & $V i_{T B V}$ & $\begin{array}{c}\text { CIx } \\
\text { Indiv }\end{array}$ & $D_{B S V}$ & $D_{B y p}$ & $D_{E L k g}$ & $D_{I L k g}$ & $S t_{B y p}$ & $V i_{B S V}$ & $V i_{T B V}$ \\
\hline$D_{B S V}$ & 0 & 1,5 & 0,6 & 0,6 & 1,4 & 0,9 & 1,2 & $D_{B S V}$ & 0 & 1,5 & 0,7 & 0,7 & 1,4 & 0,8 & 1,4 \\
\hline$D_{\text {Byp }}$ & 1,5 & 0 & 2,1 & 2 & 2,4 & 1,9 & 1,6 & $D_{B y p}$ & 1,5 & 0 & 2,2 & 2,2 & 2,7 & 1,7 & 0,6 \\
\hline$D_{E L k g}$ & 0,6 & 2,1 & 0 & 0,1 & 0,8 & 0,5 & 1 & $D_{E L k g}$ & 0,7 & 2,2 & 0 & 0 & 0,7 & 0,9 & 2 \\
\hline$D_{I L k g}$ & 0,6 & 2 & 0,1 & 0 & 0,8 & 0,5 & 1 & $D_{I L k g}$ & 0,7 & 2,2 & 0 & 0 & 0,7 & 0,9 & 2 \\
\hline$S t_{B y p}$ & 1,4 & 2,4 & 0,8 & 0,8 & 0 & 0,6 & 0,8 & $S t_{B y p}$ & 1,4 & 2,7 & 0,7 & 0,7 & 0 & 1,1 & 2,1 \\
\hline$V i_{B S V}$ & 0,9 & 1,9 & 0,5 & 0,5 & 0,6 & 0 & 0,5 & $V i_{B S V}$ & 0,8 & 1,7 & 0,9 & 0,9 & 1,1 & 0 & 1,1 \\
\hline$V i_{T B V}$ & 1,2 & 1,6 & 1 & 1 & 0,8 & 0,5 & 0 & $V \boldsymbol{i}_{T B V}$ & 1,4 & 0,6 & 2 & 2 & 2,1 & 1,1 & 0 \\
\hline
\end{tabular}

\subsection{Numerical key performance indicators}

Based on the reduced syndromes resulting from the MCS presented in the previous section, the NKPIs presented in
Section 4 are computed. The NKPIs are computed for the following specifications: a maximal false positive rate equal to $5 \%$ and a minimal true positive rate equal to $80 \%$. In practice, the true positive and false positive ratios necessary to draw the ROC curves 
Table 10

Prognostic NKPIs for fleet and individual model cases.

\begin{tabular}{|c|c|c|c|c|c|c|c|c|c|c|c|c|c|c|c|}
\hline $\begin{array}{c}D M v \\
\text { Fleet }\end{array}$ & $D_{B S V}$ & $D_{B y p}$ & $D_{E L k g}$ & $D_{I L k g}$ & $S t_{B y p}$ & $V i_{B S V}$ & $V i_{T B V}$ & $\begin{array}{c}D M v \\
\text { Indiv }\end{array}$ & $D_{B S V}$ & $D_{B y p}$ & $D_{E L k g}$ & $D_{\text {ILkg }}$ & $S t_{B y p}$ & $V i_{B S V}$ & $V i_{T B V}$ \\
\hline$D_{B S V}$ & 0.05 & - & 0.86 & 0.86 & - & - & - & $D_{B S V}$ & 0.06 & 0.06 & 1.07 & 1.07 & - & - & - \\
\hline
\end{tabular}

are computed from the healthy and faulty distributions of HIs. For example, in Fig. 11, the top left distributions are obtained via uncertainties propagation on the kriging model. The healthy distribution is the one corresponding to a magnitude equal to 0 , and the faulty distribution is the one with the highest magnitude. This faulty distribution is associated with degradation mode 1. Other similar uncertainties propagation are run for the rest of the degradation modes and other HIs to complete the set of distributions. The results are presented in the following tables for fleet and individual model cases. Tables 7 and 8 indicate that the results are better for the individual model case, which means that a monitoring particularized by the system is more efficient. Moreover, these tables indicate that of the degradation modes, seven are detectable with the required FP and TP, but as previously discussed, the most important are the critical degradation modes $\mathbf{D}_{\text {ELkg }}$ and $\mathbf{D}_{\text {ILkg }}$. This condition is widely verified because they are detectable by all three HIs.

Considering that two signatures are separable enough if the angle between them is superior to $0.5 \mathrm{rad}$, Table 9 indicates that the critical degradation modes are identifiable from the other ones. The fact that they are not identifiable from each other is irrelevant because they concern the same equipment. Table 10 shows that $\mathbf{D}_{\mathbf{B S v}}$ and $\mathbf{D}_{\mathbf{B y p}}$ have short detection margins, whereas the critical degradation modes have extremely large margins of approximately $70 \%$ of the MAM.

\section{Conclusion}

In this paper, we have proposed and implemented a novel method aimed at performing an early validation of HIs for detection and identification in design stages. This method is based on a combination of a sensitivity analysis and surrogate modeling techniques to perform a model-based computation of the HIs distributions for each degradation mode of the system. The sensitivity analysis is performed in two stages, the first is based on the Morris method, and the second is based on Sobol indices. This sensitivity analysis allows for a reduction in the number of input parameters for the kriging model and for a focus on the uncertainties quantification efforts of the most influent parameters. The method then proposes to calculate certain numerical key performance indicators to quantify the efficiency of the health indicators set, with respect to detection, identification and prognostics, before the controller implementation.

The main novelty of this paper is to import a well-known surrogate modeling technique to the domain of prognostics and health management for complex systems and, more precisely, to add the degradation parameters into the modeling process. From an industrial perspective, the benefits are threefold: health monitoring algorithm developers rely on it to provide them with data, controller designers rely on it to give them some quantified information and insurance about health indicators before the entry into service of the system and maintenance operators rely on it to provide a preview of the embedded health monitoring system capabilities in to jointly develop their maintenance strategy.

This method was applied to the monitoring of the fuel pumping unit of an aircraft engine and exhibited good results first in analyzing the sensitivity of the $\mathrm{HI}$ to each parameter and in subsequently modeling the distributions of the HIs for different magnitudes of degradations. Eventually, the computation of numerical key performance indicators allowed us to conclude that a specific model, or individual model, is more suitable for this application and that, in this case, the critical degradation modes are detectable and identifiable with a good detection margin. For future prospects, theoretical improvement can be made in the construction of the kriging model, particularly in the definition of the learning points and the estimation of the correlation parameters. On a more practical level, the method could be applied to other aircraft engine subsystems, such as the fuel metering unit.

\section{References}

[1] Patterson-Hine A, Biswas G, Aaseng G, Narasimhan S, Pattipati K. A review of diagnostic techniques for ishm applications. In: 1st Integrated systems health engineering and management forum; 2005.

[2] Roemer M, Byington CS, Kacprzynskiet GJ, Vachtsevanos J. An overview of selected prognostic technologies with reference to an integrated PHM architecture; 2007.

[3] Johnson SB, Gormley TJ, Kessler SS, Mott CD, Patterson-Hine A, Reichard KM, et al. System health management with aerospace applications. Wiley; 2011.

[4] OSA-CBM Website [On Line]. Available: 〈http://www.osacbm.org/〉.

[5] Kalgren PW, Byington CS, Roemer MJ, Watson MJ, Defining PHM, a lexical evolution of maintenance and logistics. In: IEEE autotestcon; 2006.

[6] Sheppard J, Kaufman M, Wilmer T. IEEE standards for prognostics and health management. Aerosp Electron Syst Mag 2009;24(9):34-41.

[7] Mechbal N, Vergé M, Coffignal G, Ganapathi M. Application of a combined active control and fault detection scheme to an active composite flexible structure. Mechatronics 2006;16:193-208.

[8] Chen C, Vachtsevanos G. Bearing condition prediction considering uncertainty: an interval type-2 fuzzy neural network approach. Robot Comput Integrated Manuf 2012;28(14):509-16.

[9] Saha B, Goebel K, Poll S, Christophersen J. Prognostics methods for battery health monitoring using a Bayesian framework. IEEE Trans Instrum Meas 2009;58(12):291-6.

[10] Chen J, Patton RJ. Review of parity space approaches to fault diagnosis for aerospace systems. J Guid Control Dyn 2012;17(12).

[11] Massé J, Lamoureux B, Boulet X, Prognosis and health management in system design. In: IEEE international conference on prognostics and health management, Denver; 2011.

[12] De Rocquigny E, Devictor N, Tarantola S, Mangeant F, Schwob C, Bolado-Lavin $\mathrm{R}$, et al. Uncertainty in industrial practice: a guide to quantitative uncertainty management. Wiley; 2007.

[13] Yao W, Xiaoqian C, Wencai L, van Tooren M, Guo J. Review of uncertaintybased multidisciplinary design optimization methods for aerospace vehicles. Prog Aerosp Sci 2011;47:450-79 (July).

[14] Metropolis N, Ulam S. The Monte Carlo method. J Am Stat Assoc 1949;44 (1247):335-41.

[15] Wickens TD. Elementary signal detection theory. Oxford University Press; 2002.

[16] Fawcett T. An introduction to ROC analysis. 2164 Staunton Court, Palo Alto: Institute for the Study of Learning and Expertise; 2005.

[17] Bradley AP. The use of the area under the ROC curve in the evaluation of machine learning algorithms. Pattern Recogn 1997;30(7):1145-59.

[18] Gini C. Concentration and dependency ratios. Riv Polit Econ 1997 (187):769-92.

[19] Iooss B. Revue sur l'Analyse de Sensibilité Globale de Modèles Numériques. J Soc Fran Stat 2011;152(11):3-25.

[20] Morris MD. Factorial sampling plans for preliminary computational experiments. Technometrics 1991:33(12):161-74.

[21] Sobol IM. Sensitivity estimates for nonlinear mathematical models. Math Modell Comput Exp 1993;1:407-14.

[22] Hoeffding W. A class of statistics with asymptotically normal distributions. Ann Math Stat 1948;19:293-325.

[23] Homma T, Saltelli A. Importance measures in global sensitivity analysis of non linear models. Reliab Eng Syst Saf 1996;52:1-17.

[24] Saltelli A. Making best use of model evaluations to compute sensitivity indices. Comput Phys Commun 2002;145:280-97.

[25] Marrel A, Iooss B, Laurent B, Roustant O. Calculations of Sobol indices for the Gaussian process metamodel. Reliab Eng Syst Saf 2009;94(13):742-51.

[26] Bourinet JM, FERUM 4.1 User's Guid; 2010. 
[27] Forrester AIJ, Keane AJ. Recent advances in surrogate-based optimization. Prog Aerosp Sci 2009:45(11-3):50-79.

[28] Kleijnen JPC. kriging metamodeling in simulation: a review. Eur J Oper Res 2009;192(13):707-16.

[29] Nielsen HB, Lophaven SN, Sondergaard J. DACE - A Matlab kriging Toolbox. Informatics and mathematical modelling. Lyngby - Denmark: Technical University of Denmark, DTU; 2002.

[30] Lamoureux B, Massé J-R, Mechbal N, A diagnosis methodology for the hydromechanical actuation loops in aircraft engines. In: Proceedings of the 20th Mediterranean conference on control and automation, Barcelona; 2012 972-7.

[31] Lamoureux, B, Massé JR, Mechbal N, An approach to the health monitoring of a pumping unit in an aircraft engine fuel system. In: Chez proceedings of first European conference of the prognostics and health management society, Dresden; 2012.

[32] Bouti A, Kadi DA. A state-of-the-art review of FMEA/FMECA. Int J Reliab Qual Saf Eng 1994;1(14):515-43.

[33] Lophaven SN, Nielsen HB, Søndergaard J, DACE, a matlab kriging toolbox; 2002. 